\title{
Detection of the blazar S4 0954+65 at very-high-energy with the MAGIC telescopes during an exceptionally high optical state
}

MAGIC Collaboration: M. L. Ahnen ${ }^{1}$, S. Ansoldi ${ }^{2,20}$, L. A. Antonelli ${ }^{3}$, C. Arcaro ${ }^{4}$, D. Baack ${ }^{5}$, A. Babić ${ }^{6}$, B. Banerjee ${ }^{7}$, P. Bangale ${ }^{8}$, U. Barres de Almeida ${ }^{8,9}$, J. A. Barrio ${ }^{10}$, W. Bednarek ${ }^{11}$, E. Bernardini ${ }^{4,12,23}$, R. Ch. Berse ${ }^{5}$, A. Berti ${ }^{2,22}$, W. Bhattacharyya ${ }^{12}$, A. Biland ${ }^{1}$, O. Blanch ${ }^{13}$, G. Bonnoli ${ }^{14}$, R. Carosi ${ }^{14}$, A. Carosi ${ }^{3}$, G. Ceribella ${ }^{8}$, A. Chatterjee ${ }^{7}$,

S. M. Colak ${ }^{13}$, P. Colin ${ }^{8}$, E. Colombo ${ }^{15}$, J. L. Contreras ${ }^{10}$, J. Cortina ${ }^{13}$, S. Covino ${ }^{3}$, P. Cumani ${ }^{13}$, P. Da Vela ${ }^{14}$,

F. Dazzi ${ }^{3}$, A. De Angelis 4 , B. De Lotto ${ }^{2}$, M. Delfino ${ }^{13,25}$, J. Delgado ${ }^{13}$, F. Di Pierro ${ }^{4}$, A. Domínguez ${ }^{10}$,

D. Dominis Prester ${ }^{6}$, D. Dorner ${ }^{16}$, M. Doro ${ }^{4}$, S. Einecke ${ }^{5}$, D. Elsaesser ${ }^{5}$, V. Fallah Ramazani ${ }^{17}$,

A. Fernández-Barral ${ }^{13}$, D. Fidalgo ${ }^{10}$, M. V. Fonseca ${ }^{10}$, L. Font ${ }^{18}$, C. Fruck $^{8}$, D. Galindo ${ }^{19}$, R. J. García López ${ }^{15}$, M. Garczarczyk ${ }^{12}$, M. Gaug ${ }^{18}$, P. Giammaria ${ }^{3}$, N. Godinović ${ }^{6}$, D. Gora ${ }^{12}$, D. Guberman ${ }^{13}$, D. Hadasch ${ }^{20}$, A. Hahn ${ }^{8}$,

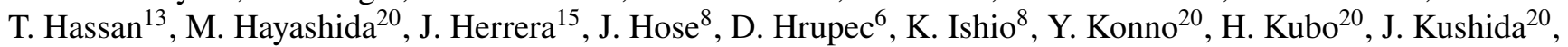
D. Kuveždić ${ }^{6}$, D. Lelas ${ }^{6}$, E. Lindfors ${ }^{17}$, S. Lombardi ${ }^{3}$, F. Longo $^{2,24}$, M. López ${ }^{10}$, C. Maggio ${ }^{18}$, P. Majumdar ${ }^{7}$, M. Makariev ${ }^{21}$, G. Maneva ${ }^{21}$, M. Manganaro ${ }^{15, \star}$, K. Mannheim $^{16}$, L. Maraschi ${ }^{3}$, M. Mariotti $^{4}$, M. Martínez ${ }^{13}$, S. Masuda ${ }^{20}$, D. Mazin ${ }^{8,20}$, K. Mielke ${ }^{5}$, M. Minev ${ }^{21}$, J. M. Miranda ${ }^{16}$, R. Mirzoyan ${ }^{8}$, A. Moralejo ${ }^{13}$, V. Moreno ${ }^{18}$, E. Moretti ${ }^{8}$, T. Nagayoshi ${ }^{20}$, V. Neustroev ${ }^{17}$, A. Niedzwiecki ${ }^{11}$, M. Nievas Rosillo ${ }^{10}$, C. Nigro ${ }^{12}$, K. Nilsson ${ }^{17}$, D. Ninci ${ }^{13}$, K. Nishijima ${ }^{20}$, K. Noda ${ }^{13}$, L. Nogués ${ }^{13}$, S. Paiano ${ }^{4}$, J. Palacio ${ }^{13}$, D. Paneque ${ }^{8}$, R. Paoletti ${ }^{14}$, J. M. Paredes ${ }^{19}$, G. Pedaletti ${ }^{12, \star}$, M. Peresano ${ }^{2}$, M. Persic ${ }^{2,26}$, P. G. Prada Moroni ${ }^{22}$, E. Prandini ${ }^{4}$, I. Puljak ${ }^{6}$, J. R. Garcia ${ }^{8}$,

I. Reichardt ${ }^{4}$, W. Rhode ${ }^{5}$, M. Ribó ${ }^{19}$, J. Rico ${ }^{13}$, C. Righi ${ }^{3}$, A. Rugliancich ${ }^{14}$, T. Saito ${ }^{20}$, K. Satalecka ${ }^{12}$, T. Schweizer ${ }^{8}$, J. Sitarek ${ }^{11,20}$, I. Šnidarić ${ }^{6}$, D. Sobczynska ${ }^{11}$, A. Stamerra ${ }^{3}$, M. Strzys ${ }^{8}$, T. Surić ${ }^{6}$, M. Takahashi2 ${ }^{20}$, L. Takalo ${ }^{17}$,

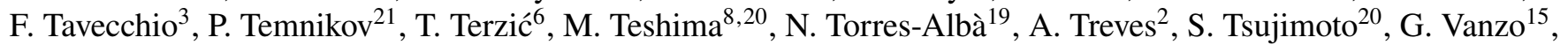
M. Vazquez Acosta ${ }^{15}$, I. Vovk $^{8}$, J. E. Ward ${ }^{13}$, M. Will ${ }^{8}$, D. Zarićc 6 , J. Becerra González ${ }^{15,27, \star}$, Y. Tanaka ${ }^{28}$, R. Ojha $^{27,29,30}$, J. Finke ${ }^{31}$ (for the Fermi-LAT Collaboration), A. Lähteenmäki ${ }^{32,33,34}$, E. Järvelä ${ }^{32,33}$, M. Tornikoski ${ }^{32}$,

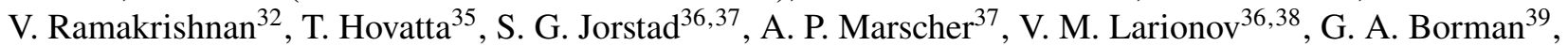
T. S. Grishina ${ }^{36}$, E. N. Kopatskaya ${ }^{36}$, L. V. Larionova ${ }^{36}$, D. A. Morozova ${ }^{36}$, S. S. Savchenko ${ }^{36}$, Yu. V. Troitskaya ${ }^{36}$, I. S. Troitsky ${ }^{36}$, A. A. Vasilyev ${ }^{36}$, I. Agudo ${ }^{40}$, S. N. Molina ${ }^{40}$, C. Casadio ${ }^{41,40}$, M. Gurwell ${ }^{42}$, M. I. Carnerero ${ }^{43}$, C. Protasio ${ }^{15,44}$, and J. A. Acosta Pulido ${ }^{15,44}$

(Affiliations can be found after the references)

Received 12 January 2018 / Accepted 19 March 2018

\section{ABSTRACT}

\begin{abstract}
Aims. The very high energy (VHE $\gtrsim 100 \mathrm{GeV}$ ) $\gamma$-ray MAGIC observations of the blazar S4 $0954+65$, were triggered by an exceptionally high flux state of emission in the optical. This blazar has a disputed redshift of $z=0.368$ or $z \geqslant 0.45$ and an uncertain classification among blazar subclasses. The exceptional source state described here makes for an excellent opportunity to understand physical processes in the jet of S4 $0954+65$ and thus contribute to its classification.

Methods. We investigated the multiwavelength (MWL) light curve and spectral energy distribution (SED) of the S4 0954+65 blazar during an enhanced state in February 2015 and have put it in context with possible emission scenarios. We collected photometric data in radio, optical, X-ray, and $\gamma$-ray. We studied both the optical polarization and the inner parsec-scale jet behavior with $43 \mathrm{GHz}$ data. Results. Observations with the MAGIC telescopes led to the first detection of S4 0954+65 at VHE. Simultaneous data with Fermi-LAT at high energy $\gamma$-ray(HE, $100 \mathrm{MeV}<E<100 \mathrm{GeV}$ ) also show a period of increased activity. Imaging at $43 \mathrm{GHz}$ reveals the emergence of a new feature in the radio jet in coincidence with the VHE flare. Simultaneous monitoring of the optical polarization angle reveals a rotation of approximately $100^{\circ}$.

Conclusions. The high emission state during the flare allows us to compile the simultaneous broadband SED and to characterize it in the scope of blazar jet emission models. The broadband spectrum can be modeled with an emission mechanism commonly invoked for flat spectrum radio quasars (FSRQs), that is, inverse Compton scattering on an external soft photon field from the dust torus, also known as external Compton. The light curve and SED phenomenology is consistent with an interpretation of a blob propagating through a helical structured magnetic field and eventually crossing a standing shock in the jet, a scenario typically applied to FSRQs and low-frequency peaked BL Lac objects (LBL).
\end{abstract}

Key words. gamma rays: galaxies - galaxies: active - BL Lacertae objects: individual: S4 0954+65

\footnotetext{
${ }^{\star}$ Corresponding authors: G. Pedaletti (giovanna . pedaletti@desy . de), M. Manganaro (manganaro@iac . es), J. Becerra González (jbecerra@iac.es).
} 


\section{Introduction}

Blazars are a subclass of active galactic nuclei (AGN) in which the relativistic jet presents a small viewing angle toward the observer and thus where relativistic effects on the observed emission are more extreme. Conventionally, blazars are subdivided in BL Lac objects and flat spectrum radio quasars (FSRQs) depending on the characteristic of their optical spectrum: while BL Lac objects are dominated by the featureless continuum emission from the jet, FSRQs show wide optical emission lines. The optical spectrum of a fraction of BL Lac objects presents features attributable to the host galaxy.

The blazar S4 0954+65 hosts a black hole of mass $M_{\mathrm{BH}} \sim 3.3 \times 10^{8} M_{\odot}$, estimated from the width of the $\mathrm{H}_{\alpha}$ line (Fan \& Cao 2004). The detection of the $\mathrm{H}_{\alpha}$ line is not confirmed by Landoni et al. (2015; see the discussion on the redshift determination) so that the mass estimation cannot be confirmed either. This blazar presents strong variability in the optical band, already well studied by Wagner et al. (1990) and by Morozova et al. (2014). Intra night variability has been found both in optical and radio wavelengths (Wagner et al. 1993). The optical high brightness state of February 2015, presented here, is however exceptional for the object, with a brightening of more than three magnitudes in the $R$-band with respect to the average monitored state ${ }^{1}$. This not only spurred many alerts in the community (see Astronomer's Telegram (ATel) \#6996, \#7001, \#7057, \#7083, \#7093; Carrasco et al. 2015; Stanek et al. 2015; Spiridonova et al. 2015; Bachev 2015; Ojha et al. 2015), but also the first and only detection of the object at very high energies (VHE, $E \gtrsim 100 \mathrm{GeV}$ ), thanks to observations by the MAGIC Telescopes. This detection by MAGIC and the multiwavelength (MWL) data collected alongside it are the focus of the present work.

The source GRO J0957+65, detected with the Energetic Gamma Ray Experiment Telescope (EGRET) telescope on board the Compton Gamma-Ray Observatory, has been associated through optical and radio observations with S4 0954+65 by Mukherjee et al. (1995). S4 0954+65 has been afterward always included in the released catalogs of sources detected by the Large Area Telescope (LAT) instrument on board the Fermi satellite (Abdo et al. 2010; Nolan et al. 2012; Ackermann et al. 2013, 2016; Acero et al. 2015; Ajello et al. 2017), with the exclusion of the bright source list released after the first three months of Fermi-LAT data integration.

The classification of the object, based on the available literature, is still unclear. In most of the ATels mentioned above S4 $0954+65$ is referenced as a FSRQ, but in most of the literature this is classified as a BL Lac object due to the small equivalent width of the emission lines in its spectrum (see, e.g., Stickel et al. 1991). Sambruna et al. (1996) classified the spectral energy distribution (SED) of S4 0954+65 as "FSRQ-like", in a sample limited to the sources with a detection from EGRET data. It indeed presents a flatter spectral index than most BL Lac objects, in both X-ray and $\gamma$-ray bands (see Raiteri er al. 1999; and references therein). Among BL Lac objects, a further phenomenological subdivision can be made based on the frequency of the synchrotron peak, ranging from optical to X-ray frequency and identifying the classes of low-, intermediate-, or high-peaked BL Lac object (LBL, IBL, HBL, respectively). Ghisellini et al. (2011) classified this object as a LBL based on the SED. When including the kinematic features from the radio jet in the classification templates,

http://users.utu.fi/kani/1m/S4_0954+65.html
Hervet et al. (2016) classify this as their kinematic class II, mostly composed of FSRQ. S4 0954+65 can thus be interpreted as a transitional object between FSRQ and classical BL Lac objects.

The most numerous extragalactic sources detected at VHE from Imaging Air Cherenkov Telescopes (IACTs), presently, belong to the HBL class. Therefore the VHE detection of an object such as S4 0954+65 provides a rare opportunity to study VHE emission conceivably produced in a different kind of environment. Indeed, while emission in HBL can mostly be satisfactorily modeled taking into account only processes in a compact feature in the jet, for FSRQs the inclusion of the interactions of such a feature with the surrounding ambient becomes of greater importance (see e.g., Tavecchio 2016). The structure of the broadband SED collected here will also be put in context with other common characteristics of a FSRQ classification, such as intrinsic brightness, peak of the synchrotron component and Compton dominance.

The question of redshift for S4 0954+65 is also still not settled, as claims of line detection in the optical spectrum are not always confirmed. The redshift of the source was first determined at $z=0.368$ by the identification of lines by Lawrence et al. (1986, 1996). Stickel et al. (1993) obtained, from different measurements, the same redshift estimate based on line identification. None of these lines were confirmed by the observations reported in Landoni et al. (2015), who instead pose a lower limit of $z \geq 0.45$. The latter results were obtained with a superior resolution spectra. At the time of the observation the magnitude in $R$-band of the object was 15.5, while it is known from variability studies that it could be even two magnitudes lower. In the following we will adopt the redshift $z=0.368$.

The outline of this paper is as follows. In Sect. 2, we present the MAGIC telescopes and the relative data set on S4 0954+65. Section 3 reviews all the MWL data that were collected during this exceptional burst, whereas Sect. 4 discusses the implication of this burst for the source state and inner jet structure. Additional information on the MAGIC data analysis, the parameters derived from the radio data, and the full dataset for X-ray data will be found in Appendix A, B, and C respectively.

\section{MAGIC observations}

The Major Atmospheric Gamma Imaging Cherenkov Telescopes (MAGIC) telescopes are an array of two IACTs located in the Island of La Palma (Spain) at an altitude of $\sim 2200 \mathrm{~m}$ asl. The system is sensitive down to an energy threshold of $E \sim 50 \mathrm{GeV}$ (Aleksić et al. 2016) for low zenith angle observations. This is of particular relevance for the monitoring of variable sources and of those that tend to exhibit a steep spectrum at VHE. The full data have been analyzed using the standard MAGIC analysis chain and the MAGIC Standard Analysis Software (MARS; Zanin et al. 2013; Aleksić et al. 2016).

The MAGIC collaboration supports a program of targets of opportunity (ToO), triggered by MWL monitoring. The ToO program was activated for observations of S4 0954+65 at the end of January 2015 after the first hints of enhanced optical state (triggered by the Tuorla monitoring in $R$-band, see Sect. 3.3). We observed the source with the MAGIC telescopes for 2 nights (MJD 57049-57050, 2015 January 27 and 28), for a total of $1 \mathrm{~h}$ high-quality dark time data, but obtained no detection. We resumed the ToO observations in February after the Tuorla monitoring revealed a very exceptional flux state, later confirmed by other monitoring programs (see Sect. 3.3). We obtained a 


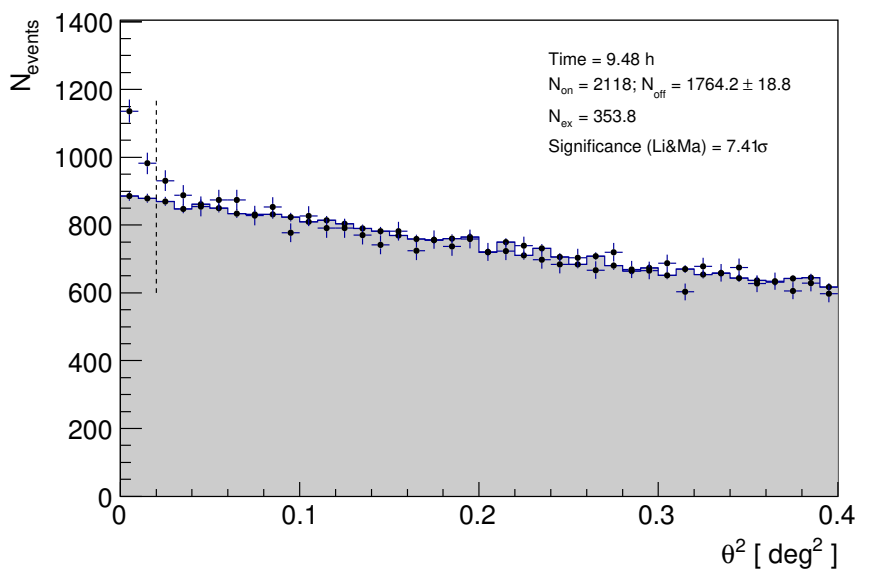

Fig. 1. Distribution of the squared angular distance $\left(\theta^{2}\right)$ between the reconstructed event direction and the nominal source direction. The filled histogram is the background estimation, obtained from sky regions within the field of view with similar detector acceptance. We show only data taken in dark condition (condition 1, see Appendix A). The standard MAGIC low energy (LE) cuts are applied to the data (see Appendix A and Table A.1). The vertical line corresponds to the optimal cut $\left(\theta^{2}=0.02 \mathrm{deg}^{2}\right)$ for point source analysis in LE cuts, used to derive significance values.

detection at a significance of $\sim 7.4 \sigma$ from observations during 2015 February 14 (MJD 57067, ATel \#7080 Mirzoyan et al. 2015). We continued observing S4 0954+65, barring adverse atmospheric conditions, until full moon days when standard MAGIC observations are not possible due to the elevated level of background light (last day of observation, with already large moonlight contamination, on 2015 March 1, MJD 57082). A detailed breakdown of the observation conditions and relative results can be found in Appendix A.

The total excess from the dark-time data is consistent with a point source emission (see Fig. 1). No other significant emission is found in the field of view apart from the one coincident with S4 0954+65 at the center.

The SED points presented in Sect. 4 below are derived for the day of the flare (MJD 57067, 2015 February 14), using only data taken in dark conditions (that allow for the lowest threshold and lowest systematic uncertainty, Appendix A). We followed the standard MAGIC unfolding procedure (Albert et al. 2007) to obtain the intrinsic spectrum.

The $\gamma$-ray emission from sources at high redshift is absorbed via photon-photon pair production on photons from the extragalactic background light (EBL; see e.g., Finke et al. 2010; Domínguez et al. 2011). S4 0954+65 redshift is assumed to be $z=0.368$. The spectral shape of the intrinsic emission, that is, after the correction for the EBL absorption, can be fitted with a simple power law

$\frac{d N}{d E}=N_{0}\left(\frac{E}{E_{0}}\right)^{-\Gamma}$,

with normalization $N_{0}=\left(13.8 \pm 2.1^{\text {stat }} \pm 1.5^{\text {sys }}\right) \times 10^{-10} \mathrm{TeV}^{-1}$ $\mathrm{cm}^{-2} \mathrm{~s}^{-1}$ at $E_{0}=0.15 \mathrm{TeV}$ and spectral index $\Gamma=3.98 \pm$ $0.67^{\text {stat }} \pm 0.15^{\text {sys }}$. The quoted systematic uncertainties are derived from the standard evaluation in MAGIC data presented by Aleksić et al. (2016). We note that the calculated systematic uncertainty on $N_{0}$ does not contain the uncertainty on the energy scale, that is about $15 \%$. The unfolded MAGIC spectrum is shown in Fig. 2. The unfolded observed spectrum, that

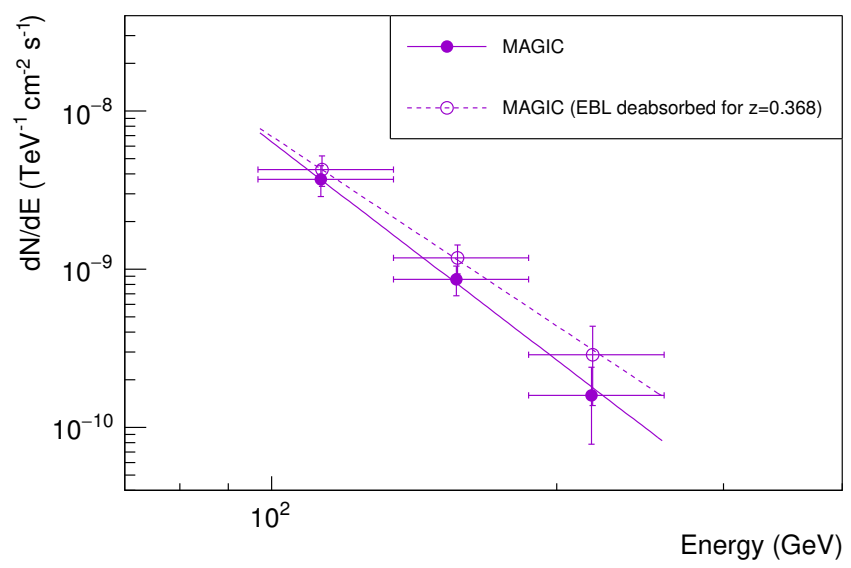

Fig. 2. Spectrum for the VHE MAGIC detection. MAGIC data are for flare night only (2015 February 14, MJD 57067.14). Violet filled circles are for the unfolded observed points, while open circles are deabsorbed for EBL absorption (EBL model by Domínguez et al. 2011). The solid line is the fit for the observed points and the dashed line is the fit for the de-absorbed ones, with details in the text.

is, without correcting for the EBL absorption, can be described also by a simple power law with $N_{0}=\left(9.9 \pm 1.5^{\text {stat }} \pm 1.1^{\text {sys }}\right) \times$ $10^{-10} \mathrm{TeV}^{-1} \mathrm{~cm}^{-2} \mathrm{~s}^{-1}$ at $E_{0}=0.15 \mathrm{TeV}$ and spectral index $\Gamma=$ $4.58 \pm 0.66^{\text {stat }} \pm 0.15^{\text {sys }}$.

\section{The multiwavelength coverage}

All the data presented in this section are collected to produce the light curves and SED, whose interpretation is later presented in Sect. 4.

\subsection{Fermi-LAT}

The LAT on board the Fermi satellite scans the entire sky every $3 \mathrm{~h}$. From the data of the first four years of operation, S4 0954+65 was detected with an average significance of $27.2 \sigma$ in the energy range from $100 \mathrm{MeV}$ to $300 \mathrm{GeV}$ as reported in the Fermi-LAT Third Source Catalog (Acero et al. 2015; 3FGL). A dedicated analysis from MJD 56952 (2014 October 22) to MJD 57208 (2015 July 05) is presented in this work. We selected Pass 8 source class events within a $10^{\circ}$ circular region centered on the position of S4 0954+65, in the energy range 0.1-500 GeV. The spectral analysis was performed through an unbinned likelihood fit, using the ScienceTools software package version v11-05-00 along with the instrument response functions P8R2_SOURCE_V6. The model of the likelihood fit includes a Galactic diffuse emission model and an isotropic component ${ }^{2}$. In addition, we included the sources in the 3FGL catalog within a $20^{\circ}$ circular region centered on S4 0954+65. The spectral indexes and fluxes of the $3 \mathrm{FGL}$ sources located within a region of $10^{\circ}$ from S4 $0954+65$ were left free to vary, while the sources in the region from $10^{\circ}$ to $20^{\circ}$ were fixed to their catalog values. The results were obtained from two iterations of maximumlikelihood analysis, after the sources with a test statistics (Mattox et al. 1996) $T S<10$ were removed. The strongest source located beyond $10^{\circ}$ from S4 $0954+65$ is at an angular distance of $10.8^{\circ}$. This source has a variability index of 42.4 in the 3FGL cata$\log$, that allows us to treat it as a nonvariable source and thus

\footnotetext{
2 Model available at https://fermi.gsfc.nasa.gov/ssc/data/ access/lat/BackgroundModels.html.
} 
to fix its spectral index and flux to the values reported in the 3FGL catalog.

The light curve was calculated in day timescale bins, modeling the source with a single power-law spectrum (as it is also described in the 3FGL). Both the flux and spectral index of S4 0954+65 were left free during the likelihood fits, while the rest of the point sources were fixed and only the diffuse Galactic and isotropic models were allowed to vary. In case of $T S<4$, an upper limit on the flux was calculated fixing the spectral index to 2.38 as given in the 3FGL catalog. The results are shown in Fig. 3. The figure also shows the light curve calculated in a 15-day bin as comparison. The light curve was obtained with the same procedure described above for the 1-day binning. During the HE flare in November 2014 (MJD 56976, ATel \#6709; Krauss 2014) the LAT spectral index is compatible with its 3FGL value of $2.38 \pm 0.04$, averaged from four years of data. Moreover, the visibility of the source by MAGIC was at an unfavorable zenith angle of $60^{\circ}$ (implying a high energy threshold). Therefore, no ToO observation was activated with MAGIC for this flare. MAGIC observations were activated later on during the strong flare on February 2015 when the LAT detected a hardening of the spectrum as shown previously by Tanaka et al. (2016) where the LAT analysis using Pass 7 reprocessed data is presented.

The spectral analysis for the MWL SED corresponds to 1-day integration centered in the MAGIC observation (MJD 57067.14, 2015 February 14). From a first likelihood fit we found the best spectral fit was a power-law spectral index of $1.87 \pm 0.09$ (significantly harder than its average 3FGL value) and was fixed in the model for the spectral points calculation. Moreover, all the sources included in the model except the diffuse Galactic and isotropic models were also fixed. The source was detected during this period with a TS of 379.7. A curved spectral model is not significantly favored in this day (TS for a log parabola fit is $T S_{\text {LP }}=380.10$ to be compared with a simple power law fit with $T S$ PWL $=379.74)$.

\subsection{Swift dataset}

The 22 multi epochs event-list obtained by the X-ray Telescope (XRT; Burrows et al. 2004) on board the Neil Gehrels Swift Observatory satellite in the period of 2014 November 17 (MJD 56978.96395) to 2015 March 11 (MJD 57092.26632) with a total exposure time of $\sim 11.12 \mathrm{~h}$ were processed using the procedure described by Fallah Ramazani et al. (2017). All these observations had been performed in photon counting (PC) mode, with an average integration time of $1.8 \mathrm{ks}$ each. The equivalent Galactic hydrogen column density is fixed to the value of $n_{H}=5.17 \times 10^{20}\left[\mathrm{~cm}^{-2}\right]$ (Kalberla et al. 2005).

The average integral photon X-ray flux $(0.3-10 \mathrm{keV})$ in this period is $1.64 \times 10^{-11} \mathrm{erg} \mathrm{cm}^{-2} \mathrm{~s}^{-1}$. The X-ray flux is peaking at MJD 57070.76523 with $F_{(0.3-10 \mathrm{keV})}=3.18 \times 10^{-11} \mathrm{erg} \mathrm{cm}^{-2} \mathrm{~s}^{-1}$ which is a factor of about two higher than the average flux of the analyzed period. The average flux outside the flare period $(2006-2015)$ is $F_{(0.3-10 \mathrm{keV})}=4.3 \times 10^{-12} \mathrm{erg} \mathrm{cm}^{-2} \mathrm{~s}^{-1}$, that we derived from a sample of XRT data comprising $25 \mathrm{X}$-ray exposures in the XRT database, not including the 22 multi epochs event-list described above. This indicates that the source was clearly in its X-ray high state during the VHE $\gamma$-ray detection. The X-ray spectral index during the analyzed period varies between $1.15 \pm 0.06 \leq \Gamma_{X} \leq 1.82 \pm 0.1$. It is notable that the softest spectral index was obtained a night prior to the VHE $\gamma$-ray flare while the spectra starts to harden after 2015 February 14 and reach its historical hardest spectra ten days after the VHE $\gamma$-ray flare. The X-ray spectra on the night before and after the VHE $\gamma$-ray flare can be well described with a power-law with spectral index of $\Gamma_{\mathrm{X}, \mathrm{Feb} .13}=1.82 \pm 0.05\left(\chi^{2} /\right.$ d.o.f. $\left.=1.024 / 41\right)$ and $\Gamma_{\mathrm{X}, \text { Feb.15 }}=1.49 \pm 0.07\left(1.025 / 24 \chi^{2} /\right.$ d.o.f. $)$ respectively. The full dataset analysis is given in Appendix C.

The Swift satellite hosts an additional instrument, the Ultraviolet/Optical Telescope (UVOT; Poole et al. 2008). The data taken during the period of interest for this work have already been presented by Tanaka et al. (2016). They follow the behavior of the optical light curve that we present below. Therefore, they are not reproduced again nor shown in Fig. 3. The UVOT bands are however important for the SED modeling presented in Sect. 4 and will therefore be included there for MJD 57067 (2015 February 14, day of the VHE detection). The dataset presented by Tanaka et al. (2016) suffers from an incorrect exposure calculation by a factor of two, related to the deadtime correction, and thus a lower reconstructed flux. We, therefore have performed a reanalysis here for the two exposures taken with UVOT on MJD 57066.76. Data reduction has been done on all the available filters $(v, b, u, w 1, m 2, w 2)$, following the standard UVOT data analysis prescriptions ${ }^{3}$. We present both exposures separately, due to the high variability in this night (e.g., for the $V$-band there is a variation of $\sim 0.3$ magnitudes in $\sim 1.5 \mathrm{~h}$ ).

\subsection{The optical domain}

Optical data were collected with: $35 \mathrm{~cm}$ KVA telescope (La Palma Island, Spain) used in the Tuorla monitoring program; $1.8 \mathrm{~m}$ Perkins telescope of Lowell Observatory (Flagstaff, Arizona); $70 \mathrm{~cm}$ telescope AZT-8 at the Crimean Astrophysical Observatory (Nauchny, Russia); $40 \mathrm{~cm}$ telescope LX-200 of St. Petersburg State University (St. Petersburg, Russia); IAC80/Camelot at the Teide Observatory (Tenerife, Spain). The data analysis from KVA was performed with the semi-automatic pipeline using the standard analysis procedures (Nilsson et al. in prep). The differential photometry was performed using the comparison star magnitudes from Villata et al. (1997). For the Perkins telescope see Jorstad et al. (2010) and references therein. The details of observations and data reductions with AZT-8 and LX-200 are given by Larionov et al. (2008). IAC80/Camelot data were automatically processed by the pipeline Redcam and calibrated astrometrically using XParallax, both available at the telescope. Instrumental magnitudes for IAC80/Camelot data were extracted using Sextractor (Bertin \& Arnouts 1996) and calibration of the source magnitude was obtained with respect to the reference stars provided by Raiteri er al. (1999).

All the telescopes mentioned above provide $R$-band photometry. We have applied the calibration of Mead et al. (1990) for all optical measurements to transform magnitudes into flux densities, and dereddened the flux according to the absorption by Schlafly \& Finkbeiner (2011). The host galaxy is not detected for this object.

From the Perkins, AZT-8+ST7 and LX-200 telescopes we collect also polarization information. In Fig. 3 we show the optical photometry data and time evolution of the fractional linear polarization and the electrical vector position angle (EVPA) in $R$-band. The EVPA measurements have been arranged such to minimize the impact of the $\pm 180^{\circ}$ ambiguity, that is, adding or subtracting $180^{\circ}$ whenever two subsequent measurements differ by more than $90^{\circ}$.

In the same timeframe of the VHE detection and the optical flare, a substantial change in the optical EVPA can be identified (see Fig. 3). The EVPA rotation starts just before the optical

https://swift.gsfc.nasa.gov/analysis/ 


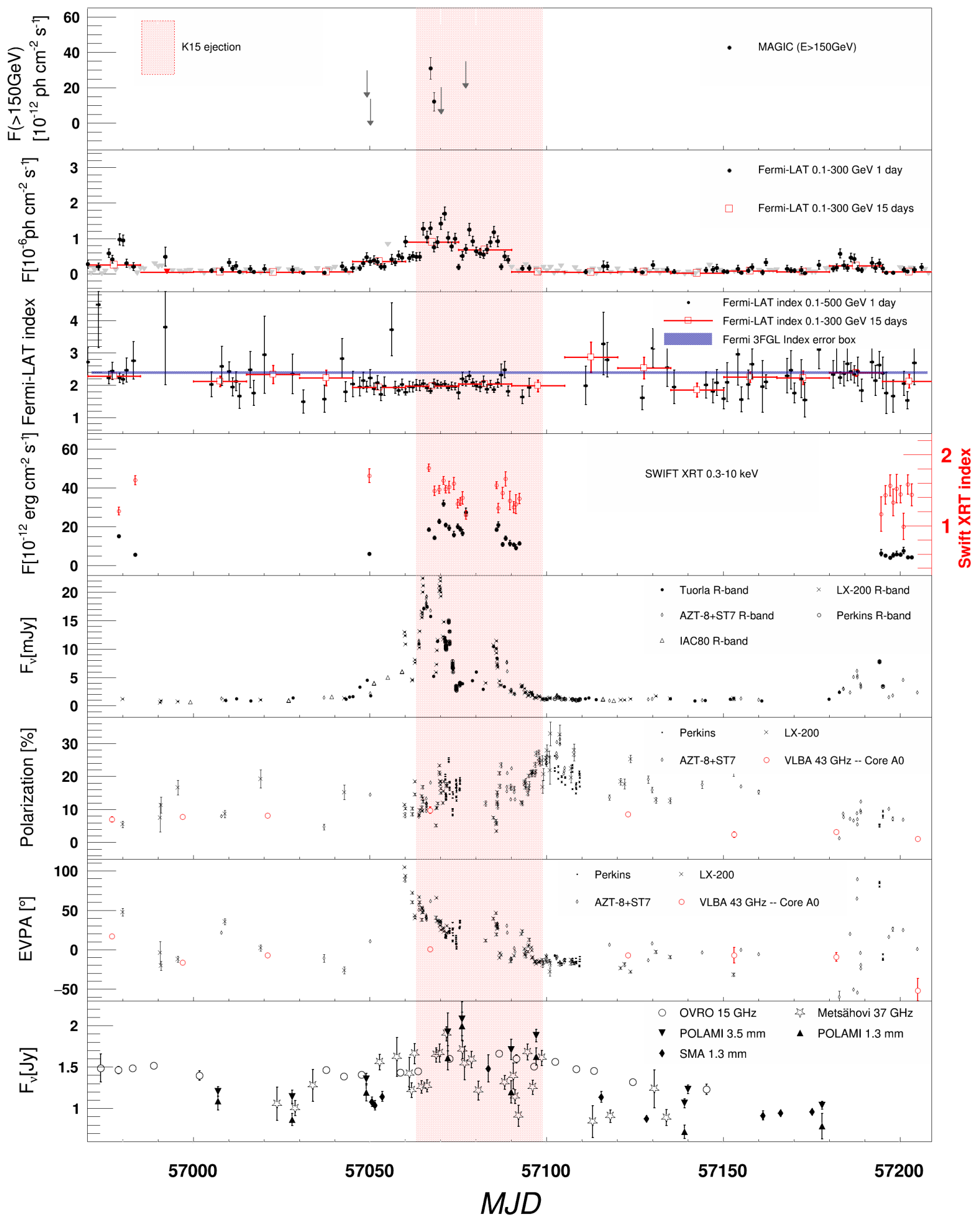

Fig. 3. MWL light curves and polarization evolution of S4 0954+65 ranging from MJD 56970 (2014 November 9) to MJD 57200 (2015 June 27). The energy range of each panel and the corresponding instrument can be found in the legend. Please refer to the text for details on the data taking and reduction for each instrument.

and VHE flare and reaches a total change of roughly $100^{\circ}$. The optical flare in February 2015 is a factor of about 3 larger in flux than the 2011 flare (see Morozova et al. 2014), that was already exceptional and concurrent with a series of $\gamma$-ray flares evident in Fermi-LAT data. During the most extreme flare in 2011, the EVPA rotated by about $300^{\circ}$. 


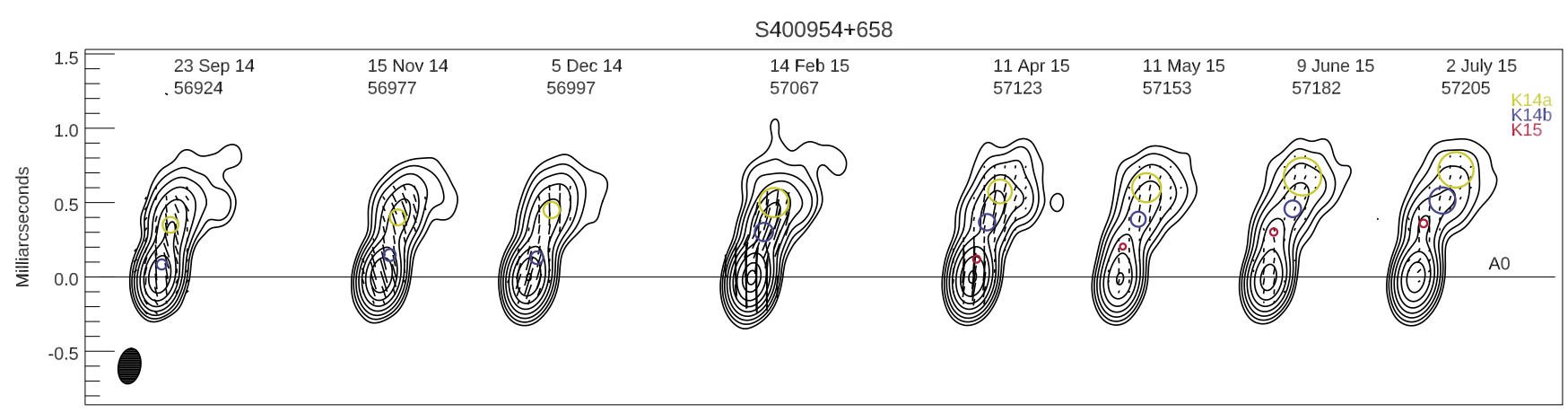

Fig. 4. Sequence of total (contours) and polarized (segments) intensity images of S4 0954+658 at $43 \mathrm{GHz}$, convolved with a beam of $0.24 \times 0.15 \mathrm{mas}^{2}$ at $\mathrm{PA}=-10^{\circ}$. The global total intensity peak is $1606 \mathrm{mJy} \mathrm{beam}^{-1}$ and the global polarized intensity peak is $104 \mathrm{mJy}^{\mathrm{beam}}{ }^{-1}$; black line segments within each image show the direction of polarization and their length is proportional to the polarized intensity. The black horizontal line indicates the position of the core, A0, and gray, blue, and red circles show the locations of knots K14a,b and K15, respectively. The size of the circles is proportional to the estimated average size in each epoch. The detailed characteristics of the knots can be found in Table 1 and B.1.

Table 1. Characteristics of the new radio knots observed from the jet of S4 0954+65.

\begin{tabular}{cccccccc}
\hline \hline Knot & $\begin{array}{c}\text { Average flux } \\
\text { mJy }\end{array}$ & $\begin{array}{c}\text { Maximum flux } \\
\text { mJy }\end{array}$ & $\begin{array}{c}\text { Average PA } \\
\operatorname{deg}\left({ }^{\circ}\right)\end{array}$ & $\begin{array}{c}\text { Average size } \\
(F W H M) \text { mas }\end{array}$ & $\begin{array}{c}\text { Proper motion } \\
\text { mas/yr }\end{array}$ & $\begin{array}{c}\text { Apparent speed } \\
\text { c }\end{array}$ & $\begin{array}{c}\text { Time of ejection } \\
\text { MJD }\end{array}$ \\
\hline K14a & $120 \pm 7$ & $286 \pm 10$ & $-17.6 \pm 2.4$ & $0.15 \pm 0.07$ & $0.55 \pm 0.04$ & $12.49 \pm 0.91$ & $56708 \pm 26$ \\
K14b & $76 \pm 25$ & $118 \pm 6$ & $-16.2 \pm 2.6$ & $0.07 \pm 0.06$ & $0.59 \pm 0.04$ & $13.47 \pm 0.86$ & $56891 \pm 15$ \\
K15 & $109 \pm 14$ & $121 \pm 5$ & $-5.9 \pm 1.9$ & $0.05 \pm 0.01$ & $1.11 \pm 0.08$ & $25.27 \pm 1.20$ & $57081 \pm 18$ \\
\hline
\end{tabular}

Notes. The evolution of parameters with the monitoring snapshots can be found in Appendix B.

\subsection{Radio and millimeter ranges}

The source S4 0954+65 was monitored at $3.5 \mathrm{~mm}(86 \mathrm{GHz})$ and $1.3 \mathrm{~mm}(229 \mathrm{GHz})$ wavelengths from the IRAM $30 \mathrm{~m}$ Millimeter Radiotelescope under the Polarimetric Monitoring of AGN at Millimeter Wavelengths ${ }^{4}$ (POLAMI) program. The program monitors the four Stokes parameters of a sample of the brightest 40 northern blazars with a cadence better than a month (see Agudo et al. 2018a,b; Thum et al. 2018). Results from the observations are presented in Fig. 3. The data reduction, calibration, and flagging procedures were described in detail by Agudo et al. (2018a), see also Agudo et al. (2010, 2014). Figure 3 includes also the $1.3 \mathrm{~mm}$ flux density data that were obtained at the Submillimeter Array (SMA) located in Hawaii. S4 0954+65 is included in an ongoing monitoring program at the SMA to determine the fluxes of compact extragalactic radio sources that can be used as calibrators at millimeter wavelengths (Gurwell et al. 2007). Observations of available potential calibrators are from time to time observed for 3-5 min, and the measured source signal strength calibrated against known standards, typically solar system objects (Titan, Uranus, Neptune, or Callisto). Data from this program are updated regularly and are available at the SMA website ${ }^{5}$. The largest flux in the considered period is at MJD 57072-57076, showing an increase of the flux between 1 and $3 \mathrm{~mm}$ wavelengths. We note, however, the lack of exactly simultaneous data to the MAGIC peak detection (MJD 57067).

S4 0954+65 is monitored monthly by the Boston University (BU) group with the Very Long Baseline Array (VLBA) at $43 \mathrm{GHz}$ within a sample of bright $\gamma$-ray blazars through the VLBA-BU-BLAZAR program ${ }^{6}$. The VLBA data are calibrated

\footnotetext{
4 http://polami.iaa.es/

5 http://sma1.sma.hawaii.edu/callist/callist.html

6 http://www.bu.edu/blazars/VLBAproject.html
}

and imaged in the same manner as discussed by Jorstad et al. $(2005,2017)$. The VLBA imaging monitoring program allows us to study the kinematics of the inner jet at pc scale. The inner jet has been monitored also for months after the VHE flare (see Fig. 4). In addition to the stable core at $\mathrm{mm}$ wavelengths (dubbed A0, see Fig. 4) it was possible to identify the emergence of three new knots whose characteristics are tabulated in Table 1. The nomenclature of the knots follows in sequential order from the beginning of the VLBA monitoring program. Previous knots characteristics can be found in Morozova et al. (2014).

Of particular interest is knot $\mathrm{K} 15$, which is very compact, with a FWHM average size of $0.05 \pm 0.01$ mas and presents the largest apparent speed of $(25.27 \pm 1.20)$ c, cf. Fig. 5. The zeroepoch separation of this knot is consistent with the VHE flare considering its 18-day uncertainty. The intensity of the core is increasing in the epoch of MJD 57067 observation, but no significant change in the core polarization can be appreciated. The detailed information on the time evolution of the radio knot can be found in Table B.1, while the polarization evolution details are shown in Table B.2. No new knot appears in November 2014, when the source was high in the HE band as observed by Fermi-LAT but without optical enhancement. The zero epochseparation from the core of knots K14a,b are not coincident with the high state in Fermi-LAT data of November 2014, but happen months before. We analyzed Fermi-LAT data for the period included within the error band for K14a,b zero epoch-separation and found no particular enhancements.

We also note the position angle of K15 with respect to the core, $\left(\mathrm{PA}=-5.9^{\circ} \pm 1.9^{\circ}\right)$. This is different than the values reconstructed from previous knots, ranging from roughly $\mathrm{PA}=-15^{\circ}$ to PA $=-25^{\circ}$ in Morozova et al. (2014), that are in turn consistent with the values for $\mathrm{K} 14 \mathrm{a}, \mathrm{b}$. The mean jet direction is at 


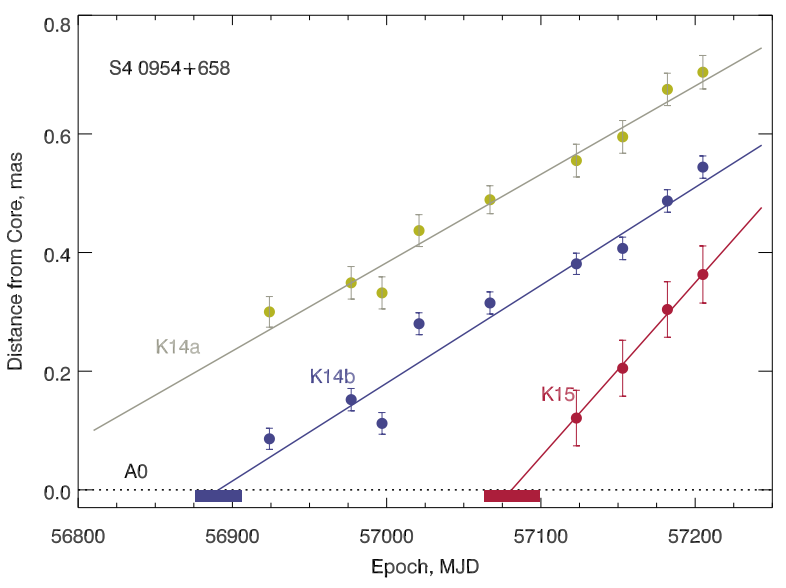

Fig. 5. Apparent distance from the radio core A0 of the new emerging knots, K14a,b and K15, as a function of time. The images from which the apparent distances are calculated can be found in Fig. 4. We note that the K15 knot presents the highest apparent speed.

$\mathrm{PA} \simeq-20^{\circ}$. A difference in PA and in apparent speed could be simply related to a small difference in the angle to the observer. However, the highest apparent speed can be used to estimate the Doppler factor, considering the upper limit to largest possible viewing angle $\theta_{\mathrm{obs}}<\arcsin \left(1 / \beta_{\text {app }}\right)$ and ultimately leading to $\delta_{\text {app }} \sim \beta_{\text {app. }}$. Applying this to the above mentioned knots (averaging the apparent speed to $\beta_{\text {app }} \sim 13 \mathrm{c}$ for K14a/b): $\theta_{\text {obs,K15 }}<2.3^{\circ}$ and $\delta_{\text {app,K15 }} \sim 25 ; \theta_{\text {obs,K14 }}<4.4^{\circ}$ and $\delta_{\text {app,K14 }} \sim 13$.

The $37 \mathrm{GHz}$ observations were made with the $13.7 \mathrm{~m}$ diameter telescope at Aalto University Metsähovi Radio Observatory. A detailed description of the data reduction and analysis is given by Teraesranta et al. (1998). The error estimate in the flux density includes contributions from the measurement RMS and the uncertainty of the absolute calibration. The S4 0954+65 observations were done as part of the regular monitoring program and the GASP-WEBT campaign. There are no strictly simultaneous $37 \mathrm{GHz}$ data to the MAGIC detection, however an increase in flux can be seen when comparing observation taken 1 day before (2015 February 13, MJD 57066.15, $F_{v}=1.27 \pm 0.07 \mathrm{Jy}$ ) and one day after the MAGIC detection (2015 February 15, MJD 57068.15, $F_{v}=1.65 \pm 0.09 \mathrm{Jy}$ ).

The Owens Valley Radio Observatory (OVRO) $40 \mathrm{~m}$ uses off-axis dual-beam optics and a cryogenic pseudo-correlation receiver with a $15.0 \mathrm{GHz}$ center frequency and $3 \mathrm{GHz}$ bandwidth. Calibration is achieved using a temperature-stable diode noise source to remove receiver gain drifts and the flux density scale is derived from observations of 3C 286 assuming the Baars et al. (1977) value of $3.44 \mathrm{Jy}$ at $15.0 \mathrm{GHz}$. The systematic uncertainty of about $5 \%$ in the flux density scale is not included in the error bars. Complete details of the reduction and calibration procedure are found in Richards et al. (2011). The long-term monitoring program at OVRO monitors the variability of this source at $15 \mathrm{GHz}$ over a longer time than what shown here. While it is obvious that the source was variable also during February 2015, it is not an exceptionally bright flux state of the source in the radio band. From a decade long monitoring, the source shows brighter levels (highest at $F_{15 \mathrm{GHz}}=2.53 \mathrm{Jy}$ ) and fainter levels (lowest at $\left.F_{15 \mathrm{GHz}}=0.85 \mathrm{Jy}\right)$.

Both 15 and $37 \mathrm{GHz}$ data seem to be in agreement with the behavior seen from $\mathrm{mm}$ wavelength data. Again we note the lack of strictly simultaneous data to the MAGIC peak detection (MJD 57067).

\section{Discussion}

The coverage of flaring states at VHE is helpful in understanding jet dynamics. We present a discussion of the SED for the day of the flare (2015 February 14). We did not attempt SED modeling for other days, for which the MAGIC data would provide only nonconstraining upper limits to emission at VHE. The day of the VHE detection is instead put in context with a longer time span behavior in the MWL dataset. However, the VHE sampling of the state is too scarce to attempt a numerical correlation study of the light curves.

\subsection{Light-curve phenomenology}

The MWL light curves of the source for all the instruments involved in the present work are reported in Fig. 3, and cover a period of seven months, from MJD 56970 (2014 November 19) to MJD 57200 (2015 June 27). The red region in Fig. 3 indicates the time window where the knot K15 was ejected in the VLBA analysis, as reported in Table 1: a time range of 36 days centered in MJD 57081 (2015 February 28). The VHE detection and the enhanced activity in the other bands are found inside the K15 ejection time window, making this event important for the understanding of the whole scenario. The spectral index at $\mathrm{HE}$ as inferred from the Fermi-LAT data is harder than the average spectral index of $\Gamma=2.38 \pm 0.04$ from the 3FGL catalog dataspan. In the presented timeframe, the X-ray emission peaks around the observation on MJD 57070.76434 (2015 February 17), with a delay with respect to the detection in VHE. The approximately 3 hours of observations in VHE in the same night did not lead to a detection (see Table A.1). However, during the period of enhanced MWL activity, there is a clear hardening of the X-ray spectrum. Hardening at both X-ray and $\gamma$-ray energies points toward the emergence of a new component in the nonthermal spectrum.

The optical band is very bright during the VHE detection, reaching peaks of more than $20 \mathrm{mJy}$ of flux density when the average behavior of the source is found around a few mJy (see the optical monitoring from Tuorla observatory). The optical emission is polarized by a fraction of $\gtrsim 10 \%$ and the polarization angle rotates by $\sim 100^{\circ}$ during the flare: Blinov et al. (2015) have shown that from a systematic monitoring (Robopol monitoring) of both $\gamma$-ray loud and $\gamma$-ray quiet sources, only the former class of object displays polarization angle rotation similar to the one seen here for S4 0954+65. Blinov et al. (2015) studied the change of EVPA as a function of time for smooth changes of $>90^{\circ}$. Requesting the same smoothness requirements, no smooth rotation of $>90^{\circ}$ can be identified in the dataset presented here, see Fig. 6. A variation of $\Delta_{\mathrm{EVPA}} \simeq 105^{\circ}$ that is not smooth can, however, be identified between MJD 57060 and MJD 57075. This variation would imply a change of the EVPA curve slope of $\Delta_{\mathrm{EVPA}} / \Delta_{t}=7 \mathrm{deg}$ day $^{-1}$, compatible with the bulk of the variations studied by Blinov et al. (2015). The rotations of the polarization angle are often physically linked to high flaring states of the objects in the $\gamma$-ray band. While individual occurrences of $\gamma$-ray flares and rotations cannot be firmly linked to each other, there is a low probability that all the occurrences are due to chance coincidence (from MonteCarlo simulations in Blinov et al. 2015). This hypothesis is still confirmed from 3 years of Robopol monitoring data in Blinov et al. (2018). Kiehlmann et al. (2017) also study whether a simple stochastic variation can account for the observed rotations in the Robopol monitoring. While their model is failing to recover all the observational characteristics in the monitoring, it also highlights a larger discrepancy from the expectations of stochastic model with 


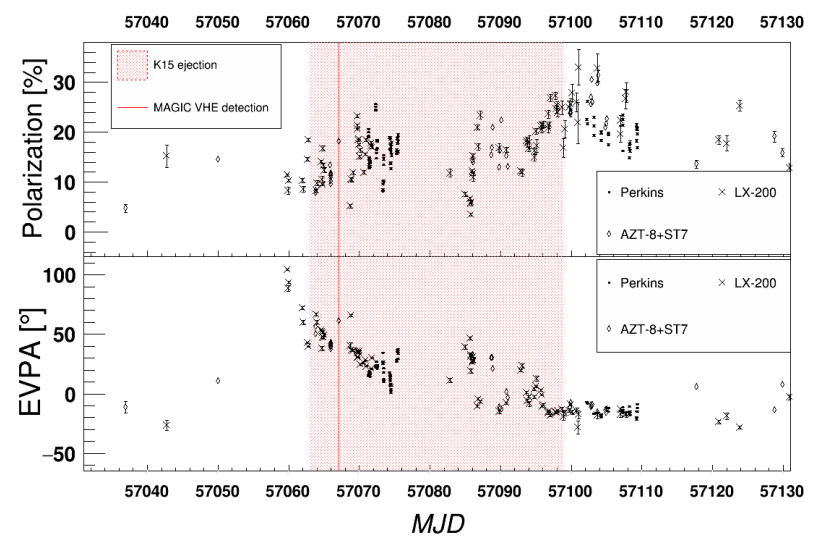

Fig. 6. Light curves for $R$-band polarimetry of S4 0954+65. Please refer to the text for details on the data taking and reduction for each instrument.

respect to the occurrence of large variations of EVPA $\left(>90^{\circ}\right)$, however not significant. Smooth variations seem also to be more firmly linked to deterministic processes and not to a random walk effect (Kiehlmann et al. 2016). Robopol monitoring data are also used in Angelakis et al. (2016), to study the difference in the amount of polarization seen on average in $\gamma$-ray loud and $\gamma$-ray quiet sources. The median fraction variability of the S4 0954+65 dataset presented here is $16.4 \%$. This value can be compared with the average $10 \%$ for the $\gamma$-ray loud subset of the Robopol monitoring and a value of $17.1 \%$ for S4 $0954+65$ computed for the observations on year 2013 and 2014. According to the interpretation by Angelakis et al. (2016), a higher fractional polarization is also expected in LSP/ISP blazars, due to the fact that in such sources the optical synchrotron emission relates to the peak synchrotron emission. Therefore, the particles associated with this emission are the most energetic, with faster cooling and thus probing a small volume of the emission region near the acceleration region, where it is expected to have a stronger ordered (helical) magnetic field, leading to higher polarization fraction.

Images at $43 \mathrm{GHz}$ show the emergence of new knots. In Morozova et al. (2014), a series of optical flares of S4 0954+65 in 2011 are studied, and the emission of knots is found correlated to the simultaneous flaring of the optical and HE bands. The maximum flux in the 2011 state is a factor of 3 lower in optical than the state presented here. The polarization fraction in this 2011 flare was similar to that seen in the present work. In Morozova et al. (2014) the chance coincidence of high optical state and knot emission has very low probability.

The phenomenology of the 2015 flare described here agrees very well with the model put forward by Marscher et al. (2008) and applied to the S4 0954+65 dataset of Morozova et al. (2014). In that model, the flare is due to a newly appearing knot accelerating at the base of the jet and propagating through an helical flow streamline. The helical streamline can be expected due to the anchoring of the accelerating flow to the rotating base of the accretion disk or black hole magnetosphere, depending on modeling. The magnetic field topology in the jet is also helical and ordered. Geometrical effects and the propagation through the helical magnetic field account for the rotation of the EVPA.

In Zhang et al. (2014), a model is proposed where the EVPA rotation is also related to the propagation through an helical magnetic field, but the streamline of propagation is not necessarily helical itself. In this model the magnitude of the swing can depend on the assumptions on the settings for the flare, specifically the magnetic field strength and orientation, the acceleration efficiency and the continuous injection of freshly accelerated particles.

The model described in Marscher et al. (2008) allows the emission at radio wavelengths in a flaring state which is not simultaneous with the VHE flare. In this scenario the radio activity could be delayed several days, even months, with respect to the VHE detection. This is expected if synchrotron self absorption is involved, and hence the emission region is located closer to the central engine than the radio core (A0 in Fig. 4). The peak of radio emission is expected to be lagging behind and appear when the disturbance has propagated further down the jet, where the absorption is not an issue. The X-ray emission peak, then, could also be delayed with respect to the optical outburst. As the X-ray emission is probably due to IC of an external soft photon field by electrons in the jet (see above), the X-ray variability traces both the accelerated particle distribution and a change in the soft photon field. This retraces similar interpretation drawn for flares of other sources where the dataset was however richer and more detailed (Marscher et al. 2008, 2010; Aleksić et al. 2014; Ahnen et al. 2017b).

\subsection{Emission model for the flare SED}

The SED of blazars are dominated by their nonthermal emission and can usually be described by two broad components. The low energy nonthermal emission is explained as synchrotron emission, while the high energy emission is most commonly modeled through inverse Compton (IC) emission, where soft photons are upscattered to $\gamma$-ray energies by electrons within the jet emitting region. The origin of the soft photon field itself can vary for different blazar subclasses. In particular, for most of the classical BL Lac objects, the VHE emission can be reasonably modeled through synchrotron self-Compton emission (SSC, see e.g., Rees 1967; Maraschi et al. 1992). Instead, for the case of FSRQs, the modeling of the emission usually requires the inclusion of external soft photon fields from, for example, the infrared dusty torus or the optical-ultraviolet emission from the broad line region (BLR) for the IC process (see e.g., Tavecchio 2016).

A broadband SED is compiled for 2015 February 14 (MJD 57067). We collect, from the MWL sample described in Sect. 3, the data closest in time to the MAGIC observation. Fermi-LAT data points are obtained from a 1-day integration centered on the MAGIC observation. The specific dates of other wavelength observations are given in the caption of Fig. 7.

Tanaka et al. (2016) model the SED of S4 0954+65 during a similar integration time as the 2015 flare studied in this work. The data shown in Fig. 7 include, in addition to what is shown by Tanaka et al. (2016), the VHE data from the MAGIC observation, the AZT-8+ST7 and POLAMI data. Moreover, the Fermi-LAT data are reanalyzed as described in Sect. 3 to be centered at the MAGIC observation time and benefit from the latest Fermi-LAT Pass 8 . The Swift-XRT and Swift-UVOT data are also reanalyzed for this work.

Tanaka et al. (2016) report that a SSC modeling of the data is challenging, requiring very low magnetic field $(B \sim 1 \mu \mathrm{G}$ in contrast to the $B \sim 1 G$ expected in blazar jet components). Alternatively, an External Compton (EC) modeling was able to reproduce the data. In their model, the soft photon field for the EC model was the dusty torus from the source. In Fig. 7, we plot the model from Tanaka et al. (2016). This model reproduces the Fermi-LAT and MAGIC data, although their paper did not 


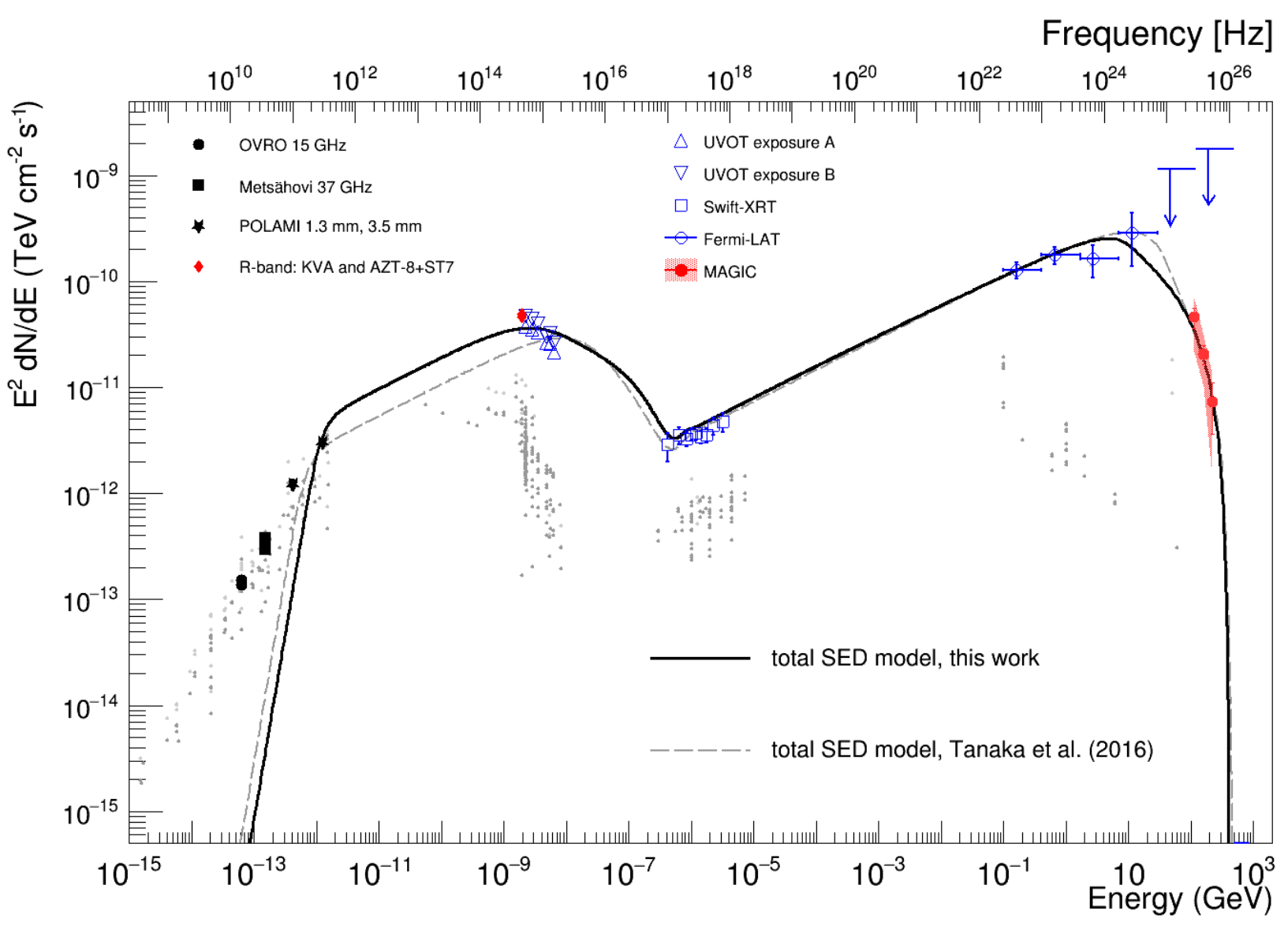

Fig. 7. Spectral energy distribution for the VHE MAGIC detection. Red symbols are strictly simultaneous to the VHE detection, blue symbols are for data taken during the same day and black symbols are for the closest observations. MAGIC spectral data (red circles) are for flare night only (2015 February 14, MJD 57067.14). Red filled circles are for the unfolded observed data points. The red shaded band shows the region of additional systematic uncertainty. Fermi-LAT data are the Pass 8 data for 2015 February 14 (1-day integration centered on the MAGIC observation, blue squares). Swift-XRT data are for 2015 February 13 (MJD 57066.70992, blue squares). Swift-UVOT data are given for the two separate exposure taken on 2015 February 13 (MJD 57066.76, blue triangles and dark blue triangles). $R$-band data are for 14th Feb (Tuorla, MJD 57067.16375 and AZT-8+ST7 MJD 57067.1, red diamonds). POLAMI data are for the 18th February (MJD 57071.5, black stars at 100 and $300 \mathrm{GHz}$ ). OVRO data are for 2015 February 10 and 19 (black circles at $15 \mathrm{GHz}$ ). Metsähovi for 2015 February 13 and 15 (MJD 57066.15, MJD 57068.68, black squares at $37 \mathrm{GHz}$ ). The gray data are for NED (light) and SSDC (dark) SED historical data points. The model from Tanaka et al. (2016; gray dashed curve) as well as the model presented here (black solide curve) include an emission component from synchrotron plus inverse Compton on a dusty torus (see text for details and Table 2 for the values of the physical parameters). The effect of the EBL attenuation is included in the modeling using the model by Finke et al. (2010) and a redshift of $z=0.368$.

include any MAGIC data. However, the model fails to reproduce properly the optical observations. Such underestimation at optical frequencies in the model of Tanaka et al. (2016) is driven by a misreconstruction of the UVOT fluxes, explained in Sect. 3. With the reanalyzed UVOT dataset presented here, we use a new model, using the same code and most of the same assumptions as in Tanaka et al. (2016), including a redshift of $z=0.368$. The code is explained in detail in Finke et al. (2008) and Dermer et al. (2009). We note that the presented SED model curves already include the effect of EBL absorption, meaning that the intrinsic emission is absorbed according to the EBL model by Finke et al. (2010). The new EC model provides a good description of the MWL data and is shown in Fig. 7. The parameters of both models are reported in Table 2 . The break in the underlying electron population is similar to what expected by classical cooling, with the slope of the electron distribution before of the break $\left(s_{1}\right)$ and after the break $\left(s_{2}\right)$ differing by $s_{2}-s_{1}=1.2$. We also tested a model with a larger break $\left(s_{2}-s_{1}=1.6\right)$ and longer variability timescale $\left(10^{5} \mathrm{~s}\right)$ which was better able to reproduce the $U V$ band data. However, the larger $s_{2}-s_{1}$ disagrees with expectations from synchro-Compton losses. Further, this model underestimates the MAGIC data and overestimates the emission at $1 \mathrm{~mm}$. We therefore do not present this model here. The use of VHE spectral information is crucial to model the falling part of the high energy peak of the blazars SEDs, which is crucial to constrain the most energetic electrons within the leptonic framework scenario (SSC and EC models).

As mentioned in the introduction, the classification of a blazar can be aided by the study of its SED characteristics. According to the SED model presented above, the peak of the synchrotron emission is at $v_{\text {syn }} \sim 8 \times 10^{14} \mathrm{~Hz}$, making it an intermediate synchrotron peaked BL Lac object

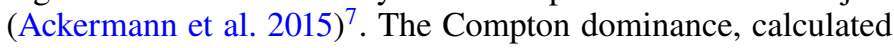
comparing the luminosity at the peak of the synchrotron emission to that of the IC peak, is $L_{\mathrm{IC}} / L_{\mathrm{syn}} \sim 7$. Such Compton dominance value is at least 3.5 times the values obtained by Finke (2013) for long-term blazar studies.

\footnotetext{
7 Intermediate-synchrotron-peaked blazar (ISP) are defined with restframe synchrotron peak frequencies of $10^{14} \mathrm{~Hz}<v_{\mathrm{syn}}<10^{15} \mathrm{~Hz}$.
} 
Table 2. SED model parameters.

\begin{tabular}{|c|c|c|c|}
\hline Parameter & Symbol & $\begin{array}{c}\text { Model A } \\
\text { Tanaka et al. (2016) }\end{array}$ & $\begin{array}{l}\text { Model B } \\
\text { This work }\end{array}$ \\
\hline Redshift & $z$ & \multicolumn{2}{|l|}{0.368} \\
\hline Bulk Lorentz factor & $\Gamma$ & 30 & 35 \\
\hline Doppler factor & $\delta_{D}$ & 30 & 35 \\
\hline Variability timescale $[\mathrm{s}]$ & $t_{v}$ & $1.0 \times 10^{5}$ & $4 \times 10^{4}$ \\
\hline Comoving radius of blob $[\mathrm{cm}]$ & $R_{b}^{\prime}$ & $6.6 \times 10^{16}$ & $3.0 \times 10^{16}$ \\
\hline Magnetic field [G] & $\stackrel{b}{B}$ & 0.6 & 0.4 \\
\hline Low-energy electron spectral index & $s_{1}$ & 2.4 & 2.4 \\
\hline High-energy electron spectral index & $s_{2}$ & 4.5 & 3.6 \\
\hline Minimum electron Lorentz factor & $\gamma_{\min }^{\prime}$ & 1.0 & 1.0 \\
\hline Break electron Lorentz factor & $\gamma_{\mathrm{brk}}^{\prime}$ & $8.0 \times 10^{3}$ & $4.0 \times 10^{3}$ \\
\hline Maximum electron Lorentz factor & $\gamma_{\max }^{\prime \prime}$ & $2.0 \times 10^{4}$ & $4.0 \times 10^{4}$ \\
\hline Black hole mass $\left[M_{\odot}\right]$ & $M_{\mathrm{BH}}$ & \multicolumn{2}{|l|}{$3.4 \times 10^{8}$} \\
\hline Disk luminosity [ $\mathrm{erg} \mathrm{s}^{-1}$ ] & $L_{\text {disk }}$ & \multicolumn{2}{|l|}{$3.0 \times 10^{43}$} \\
\hline Inner disk radius $\left[R_{g}\right]$ & $R_{\text {in }}$ & \multicolumn{2}{|l|}{6.0} \\
\hline Seed photon source energy density $\left[\mathrm{erg} \mathrm{cm}^{-3}\right]$ & $u_{\text {seed }}$ & $2.4 \times 10^{-4}$ & $4.4 \times 10^{-5}$ \\
\hline Seed photon source photon energy $\left[m_{e} c^{2}\right.$ units] & $\epsilon_{\text {seed }}$ & $7.5 \times 10^{-7}$ & $5 \times 10^{-7}$ \\
\hline Dust Torus luminosity $\left[\mathrm{erg} \mathrm{s}^{-1}\right]$ & $L_{\text {dust }}$ & $3.9 \times 10^{42}$ & $1.5 \times 10^{42}$ \\
\hline Dust Torus radius $[\mathrm{cm}]$ & $R_{\text {dust }}$ & $2.1 \times 10^{17}$ & $6.1 \times 10^{17}$ \\
\hline Dust temperature $[\mathrm{K}]$ & $T_{\text {dust }}$ & 1500 & 1000 \\
\hline Jet power in magnetic field [erg s${ }^{-1}$ ] & $P_{j, B}$ & $1.0 \times 10^{46}$ & $1.4 \times 10^{45}$ \\
\hline Jet power in electrons $\left[\mathrm{erg} \mathrm{s}^{-1}\right]$ & $P_{j, e}$ & $1.1 \times 10^{45}$ & $6.6 \times 10^{45}$ \\
\hline
\end{tabular}

\section{Conclusions}

The census of extragalactic objects that present VHE emission is still limited. We present here the first detection at VHE of the blazar S4 0954+65 obtained through observations with the MAGIC Telescopes. The observations were conducted during an exceptional flare of the source in February 2015, originally identified in the optical band. We collected MWL simultaneous data to better characterize the state of the source.

The HE emission is also found in elevated state from the analysis of Fermi-LAT data, which reveal the hardest state of the HE emission to be concurrent with the detection at VHE. The X-ray emission peak is delayed by few days with respect to the VHE detection and shows a trend of spectral hardening during the period presented here. The radio and $\mathrm{mm}$ wavelength emission reveal a moderate elevation of the flux, that is however not exceptional in the long term behavior of the source.

The source is classified in the literature as a BL Lac, but we have shown here that it presents similarities with the FSRQ class. Results from the monitoring of optical polarization and $43 \mathrm{GHz}$ jet component analysis were compared to archival observation of S4 0954+65 and of statistical behaviour of other sources. Three main measurements were considered: the day of the VHE detection of S4 0954+65 is included in the error box for the zero epoch separation of knot K15; the optical polarization fraction is increasing in the same period; a rotation of optical EVPA of $\sim 100^{\circ}$ can be identified, also in the same period, possibly related to the helical structure of the magnetic field in the acceleration region. We discuss how these measurements point to a common behavior with ISP/LSP sources. Both, the best emission model (EC on dust torus) and the MWL light curve behavior show points of contact with other sources that are either clear FSRQ (like PKS 1510-089) or are transitional objects (like BL Lac itself). This is also supported from the moderate
Compton dominance in the SED model presented here and the fact that the synchtrotron peak show that the source can be classified as ISP source. The work presented here reiterates the importance of VHE $\gamma$-ray and detailed MWL studies of blazars during different flux states to test their intrinsic characteristics and shed light on the physical processes taking place within their jets.

Acknowledgements. We would like to thank the Instituto de Astrofísica de Canarias for the excellent working conditions at the Observatorio del Roque de los Muchachos in La Palma. The financial support of the German BMBF and MPG, the Italian INFN and INAF, the Swiss National Fund SNF, the ERDF under the Spanish MINECO (FPA2015-69818-P, FPA2012-36668, FPA2015-68378-P, FPA2015-69210-C6-2-R, FPA2015-69210C6-4-R, FPA2015-69210-C6-6-R, AYA2015-71042-P, AYA2016-76012-C3-1-P, ESP2015-71662-C2-2-P, CSD2009-00064), and the Japanese JSPS and MEXT is gratefully acknowledged. This work was also supported by the Spanish Centro de Excelencia "Severo Ochoa" SEV-2012-0234 and SEV-2015-0548, and Unidad de Excelencia "María de Maeztu" MDM-2014-0369, by the Croatian Science Foundation (HrZZ) Project IP-2016-06-9782 and the University of Rijeka Project 13.12.1.3.02, by the DFG Collaborative Research Centers SFB823/C4 and SFB876/C3, the Polish National Research Centre grant UMO-2016/22/M/ST9/00382 and by the Brazilian MCTIC, CNPq, and FAPERJ. The Fermi LAT Collaboration acknowledges generous ongoing support from a number of agencies and institutes that have supported both the development and the operation of the LAT as well as scientific data analysis. These include the National Aeronautics and Space Administration and the Department of Energy in the United States, the Commissariat à l'Energie Atomique and the Centre National de la Recherche Scientifique/Institut National de Physique Nucléaire et de Physique des Particules in France, the Agenzia Spaziale Italiana and the Istituto Nazionale di Fisica Nucleare in Italy, the Ministry of Education, Culture, Sports, Science and Technology (MEXT), High Energy Accelerator Research Organization (KEK), and Japan Aerospace Exploration Agency (JAXA) in Japan, and the K. A. Wallenberg Foundation, the Swedish Research Council and the Swedish National Space Board in Sweden. This work performed in part under DOE Contract DE-AC02-76SF00515. This research has made use of the NASA/IPAC Extragalactic Database (NED), which is operated by the Jet Propulsion Laboratory, California Institute of Technology, under contract with the National Aeronautics and Space Administration. Part of this work 
is based on archival data, software, or online services provided by the Space Science Data Center - ASI. The OVRO 40-m monitoring program is supported in part by NASA grants NNX08AW31G, NNX11A043G, and NNX14AQ89G, and NSF grants AST-0808050 and AST-1109911 The research at Boston University was supported by NASA Fermi Guest Investigator program grant 80NSSC17K0694 and US National Science Foundation grant AST-1615796. The VLBA is an instrument of the Long Baseline Observatory. The Long Baseline Observatory is a facility of the National Science Foundation operated by Associated Universities, Inc. This paper is partly based on observations carried out with the IRAM $30 \mathrm{~m}$ Telescope. IRAM is supported by INSU/CNRS (France), MPG (Germany) and IGN (Spain). IA acknowledges support by a Ramón y Cajal grant of the Ministerio de Economía, Industria y Competitividad (MINECO) of Spain. The research at the IAA-CSIC was supported in part by the MINECO through grants AYA2016-80889-P, AYA2013-40825-P, and AYA2010-14844, and by the regional government of Andalucía through grant P09-FQM-4784. St. Petersburg University team acknowledges support from Russian Science Foundation grant 17-12-01029. The Submillimeter Array is a joint project between the Smithsonian Astrophysical Observatory and the Academia Sinica Institute of Astronomy and Astrophysics and is funded by the Smithsonian Institution and the Academia Sinica.

\section{References}

Abdo, A. A., Ackermann, M., Ajello, M., et al. 2010, ApJ, 188, 405 Acero, F., Ackermann, M., Ajello, M., et al. 2015, ApJS, 218, 23 Ackermann, M., Ajello, M., Allafort, A., et al. 2013, ApJS, 209, 34 Ackermann, M., Ajello, M., Atwood, W. B., et al. 2015, ApJ, 810, 14 Ackermann, M., Ajello, M., Atwood, W. B., et al. 2016, ApJS, 222, 5

Agudo, I., Thum, C., Wiesemeyer, H., \& Krichbaum, T. P. 2010, ApJS, 189,1

Agudo, I., Thum, C., Gómez, J. L., \& Wiesemeyer, H. 2014, A\&A, 566, A59

Agudo, I., Thum, C., Molina, S. N., et al. 2018a, MNRAS, 474, 1427

Agudo, I., Thum, C., Ramakrishnan, V., et al. 2018b, MNRAS, 473, 1850

Ahnen, M. L., Ansoldi, S., Antonelli, A., et al. 2017a, Astropart. Phys., 94, 29

Ahnen, M. L., Ansoldi, S., Antonelli, A., et al. 2017b, A\&A, 603, A29

Ajello, M., Atwood, W. B., Baldini, L., et al. 2017, ApJS, 232, 18

Albert, J., Aliu, E., Anderhub, H., et al. 2007, NIMPA, 583, 494

Aleksić, J., Ansoldi, S., Antonelli, L. A., et al. 2014, A\&A, 569, A46

Aleksić, J., Ansoldi, S., Antonelli, L. A., et al. 2016, Astropart. Phys., 72, 76

Angelakis, E., Hovatta, T., Blinov, D., et al. 2016, MNRAS, 463, 3365

Baars J. W. M., Genzel, R., Pauliny-Toth, I. I. K., \& Witzel, A. 1977, A\&A, 61,

Bachev, R. 2015, ATel, 7083

Bertin, E., \& Arnouts, S. 1996, A\&AS, 117, 393

Blinov, D., Pavlidou, V., Papadakis, I., et al. 2015, MNRAS, 453, 1669

Blinov, D., Pavlidou, V., Papadakis, I., et al. 2018, MNRAS, 474, 1296

Burrows, D. N., Hill, J. E., Nousek, J. A., et al. 2004, Proc. SPIE, 5165, 201

Carrasco, L., Miramon, J., Porras, A., et al. 2015, ATel, 6996

Dermer, C. D., Finke, J. D., Krug, H., \& Böttcher, M. 2009, ApJ, 692, 32

Domínguez, A., Primack, J. R., Rosario, D. J., et al. 2011, MNRAS, 410, 2556

Fallah Ramazani, V., Lindfors, E., \& Nilsson, K. 2017, A\&A 608, A68

Fan, Z.-H., \& Cao, X. 2004, ApJ, 602, 103

Finke, J. D. 2013, ApJ, 763, 134

Finke, J. D., Dermer, C. D., \& Böttcher, M. 2008, ApJ, 686, 181

Finke, J. D., Razzaque, S., \& Dermer, C. D. 2010, ApJ, 712, 238

Fruck, C., \& Gaug, M. 2015, Eur. Phys. J. Web Conf., 89, 02003

Ghisellini, G., Tavecchio, F., Foschini, L., \& Ghirlanda, G. 2011, MNRAS, 414 2674

Gurwell, M. A., Peck, A. B., Hostler, S. R., Darrah, M. R., \& Katz, C. A. 2007, in From Z-Machines to ALMA: (Sub)Millimeter Spectroscopy of Galaxies, ASP Conf. Ser., 375, 234

Healey, S. E., Romani, R. W., Taylor, G. B., et al. 2007, ApJS, 171, 61

Hervet, O., Boisson, C., \& Sol, H. 2016, A\&A, 592, A22

Impey, C. D., Lawrence, C. R., \& Tapia, S. 1991, ApJ, 375, 46I

Jorstad, S. G., Marscher, A. P., Lister, M. L., et al. 2005, AJ, 130, 1418

Jorstad, S. G., Marscher, A. P., Larionov, V. M., et al. 2010, ApJ, 715, 362

Jorstad, S. G., Marscher, A. P., Morozova, D. A., et al. 2017, ApJ, 846, 98

Kalberla, P. M. W., Burton, W. B., Hartmann, D., et al. 2005, A\&A, 440, 775

Kiehlmann, S., Savolainen, T., Jorstad, S. G., et al. 2016, A\&A, 590, A10

Kiehlmann, S., Blinov, D., Pearson, T. J., Liodakis, I. 2017, MNRAS, 472, 3589

Krauss, F. (Fermi-LAT Collaboration) 2014, ATel, 6709

Landoni, M., Falomo, R., Treves, A., Scarpa, R., \& Reverte Payà, D. 2015, AJ, 150,181

Larionov, V. M., Jorstad, S. G., Marscher, A. P., et al. 2008, A\&A, 492, 389

Lawrence, C. R., Pearson, T. J., Readhead, A. C. S., \& Unwin, S. C. 1986, AJ 91,494
Lawrence, C. R., Zucker, J. R., Readhead, A. C. S., et al. 1996, ApJS, 107, 541 Li, T.-P., \& Ma, Y.-Q. 1983, ApJ, 272, 317

Maraschi, L. 1992, ApJ, 397, L5

Marscher, A. P., Jorstad, S. G., D’Arcangelo, F. D., et al. 2008, Nature, 452, 966

Marscher, A. P., Jorstad, S. G., Larionov, V. M., et al. 2010, ApJ, 710, L126

Mattox, J. R., Bertsch, D. L., Chiang, J., et al. 1996, ApJ, 461, 396

Mazin, D., \& Göbel, F. 2007, ApJ, 655, L13

Mead, A. R. G., Ballard, K. R., Brand, P. W. J. L., et al. 1990, A\&AS, 83, 183

Mirzoyan, R. (MAGIC Collaboration) 2015, ATel, 7080

Morozova, D. A., Larionov, V. M., Troitsky, I. S., et al. 2014, AJ, 148, 42

Mukherjee, R., Aller, H. D., Aller, M. F., et al. 1995, ApJ, 445, 189

Nolan, P. L., Abdo, A. A., Ackermann, M., et al. 2012, ApJS, 199, 31

Ojha, R., Carpenter, B., \& Tanaka, Y. (Fermi-LAT Collaboration) 2015, ATel, 7093

Poole, T. S., Breeveld, A. A., Page, M. J., et al. 2008, MNRAS, 383, 627

Prandini, E., Bonnoli, G., Maraschi, L., et al. 2010, MNRAS, 405, L76

Raiteri, C. M., Villata, M., Tosti, G., et al. 1999, A\&A, 352, 19

Rees, M. J. 1967, MNRAS, 137, 429

Richards, J. L., Max-Moerbeck, W., Pavlidou, V., et al. 2011, ApJS, 194, 29

Rolke, W. A., López, A. M., \& Conrad, J. 2005, NIMPA, 551, 493

Sambruna, R. M., Maraschi, L., \& Urry, C. M. 1996, ApJ, 463, 444

Schlafly, E. F., \& Finkbeiner, D. P. 2011, ApJ, 737, 103

Spiridonova, O. I., Vlasyuk, V. V., Moskvitin, A. S., et al. 2015, ATel, 7057

Stanek, K. Z., Danilet, A. B., Holoien, T. W.-S., et al. 2015, ATel, 7001

Stickel, M., Padovani, P., Urry, C. M., Fried, J. W., \& Kuehr, H. 1991, ApJ, 374 431

Stickel M., Fried, J. W., \& Kuhr, H. 1993, A\&AS, 98, 393

Tanaka, Y. T., Becerra G., J., Itoh, R., et al. 2016, PASJ, 68, 51

Tavecchio, F. 2017, AIP Conf. Proc., 1792, 020007

Teraesranta, H., Tornikoski, M., Mujunen, A., et al. 1998, A\&AS, 132, 305

Thum, C., Agudo, I., Molina, S. N., et al. 2018, MNRAS, 473, 2506

Villata, M., Raiteri, C. M., Ghisellini, G., et al. 1997, A\&AS, 121, 119

Wagner, S., Sanchez-Pons, F., Quirrenbach, A., \& Witzel, A. 1990, A\&A, 235, 1

Wagner, S. J., Witzel, A., Krichbaum, T. P., et al., 1993, A\&A, 271, 344

Zanin, R., Carmona, E., Sitarek, J., et al. 2013, Proc. of 33rd ICRC (Rio de Janeiro, Brazil), 773

Zhang, H., Cheng, X., \& Böttcher, M. 2014, ApJ, 789, 66

1 ETH Zurich, 8093 Zurich, Switzerland

2 Università di Udine, and INFN Trieste, 33100 Udine, Italy

3 National Institute for Astrophysics (INAF), 00136 Rome, Italy

4 Università di Padova and INFN, 35131 Padova, Italy

5 Technische Universität Dortmund, 44221 Dortmund, Germany

6 Croatian MAGIC Consortium: University of Rijeka, 51000 Rijeka, University of Split - FESB, 21000 Split, University of Zagreb FER, 10000 Zagreb, University of Osijek, 31000 Osijek and Rudjer Boskovic Institute, 10000 Zagreb, Croatia

7 Saha Institute of Nuclear Physics, HBNI, 1/AF Bidhannagar, Salt Lake, Sector-1, Kolkata 700064, India

8 Max-Planck-Institut für Physik, 80805 München, Germany

9 now at Centro Brasileiro de Pesquisas Físicas (CBPF), 22290-180 URCA, Rio de Janeiro (RJ), Brasil

10 Unidad de Partículas y Cosmología (UPARCOS), Universidad Complutense, 28040 Madrid, Spain

11 University of Łódź, Department of Astrophysics, 90236 Łódź, Poland

12 Deutsches Elektronen-Synchrotron (DESY), 15738 Zeuthen, Germany

13 Institut de Física d'Altes Energies (IFAE), The Barcelona Institute of Science and Technology (BIST), 08193 Bellaterra (Barcelona), Spain

14 Università di Siena and INFN Pisa, 53100 Siena, Italy

15 Instituto de Astrofísica de Canarias, 38200 La Laguna, and Universidad de La Laguna, Departamento Astrofísica, 38206 La Laguna, Tenerife, Spain

16 Universität Würzburg, 97074 Würzburg, Germany

17 Finnish MAGIC Consortium: Tuorla Observatory and Finnish Centre of Astronomy with ESO (FINCA), University of Turku, Vaisalantie 20, 21500 Piikkiö, Astronomy Division, University of Oulu, 90014 University of Oulu, Finland

18 Departament de Física, and CERES-IEEC, Universitat Autónoma de Barcelona, 08193 Bellaterra, Spain 
19 Universitat de Barcelona, ICC, IEEC-UB, 08028 Barcelona, Spain

20 Japanese MAGIC Consortium: ICRR, The University of Tokyo, $277-$ 8582 Chiba, Japan; Department of Physics, Kyoto University, 6068502 Kyoto, Japan; Tokai University, 259-1292 Kanagawa, Japan; The University of Tokushima, 770-8502 Tokushima, Japan

${ }^{21}$ Institute for Nuclear Research and Nuclear Energy, Bulgarian Academy of Sciences, 1784 Sofia, Bulgaria

${ }^{22}$ Università di Pisa, and INFN Pisa, 56126 Pisa, Italy

${ }^{23}$ Humboldt University of Berlin, Institut für Physik 12489 Berlin, Germany

${ }^{24}$ Dipartimento di Fisica, Università di Trieste, 34127 Trieste, Italy

25 Port d'Informació Científica (PIC) 08193 Bellaterra (Barcelona), Spain

${ }^{26}$ INAF-Trieste and Deptartment of Physics and Astronomy, University of Bologna, Italy

27 NASA Goddard Space Flight Center, Greenbelt, MD 20771, USA and Department of Physics and Department of Astronomy, University of Maryland, College Park, MD 20742, USA

${ }_{28}$ Hiroshima Astrophysical Science Center, Hiroshima, Japan

29 University of Maryland, Baltimore County, USA

30 The Catholic University of America, Washington DC, USA
31 Space Science Division, NRL, Washington DC, USA

32 Aalto University Metsahovi Radio Observatory, Finland

33 Aalto University Department of Electronics and Nanoengineering, Finland

34 Tartu Observatory, Estonia

35 Tuorla Observatory, University of Turku, Väisäläntie 20, 21500 Piikkiö, Finland

36 Astronomical Institute, St. Petersburg State University, Russia

37 Institute for Astrophysical Research, Boston University, USA

${ }^{38}$ Pulkovo Observatory, St. Petersburg, Russia

39 Crimean Astrophysical Observatory, P/O Nauchny, 298409, Crimea

40 Instituto de Astrofísica de Andalucía (CSIC), Apartado 3004, 18080 Granada, Spain

${ }^{41}$ Max-Planck-Institut für Radioastronomie, Auf dem Hügel, 69, 53121, Bonn, Germany

42 Harvard-Smithsonian Center for Astrophysics, Cambridge, MA USA

43 INAF, Osservatorio Astrofisico di Torino, via Osservatorio 20, 10025, Pino Torinese, Italy

${ }^{4}$ Departamento de Astrofisica, Universidad de La Laguna, La Laguna, 38205 Tenerife, Spain 


\section{Appendix A: Additional information on MAGIC data reduction}

Table A.1. MAGIC data summary for the observation of S4 0954+65 from 2015 January 27 to 2015 March 1.

\begin{tabular}{|c|c|c|c|c|}
\hline $\begin{array}{c}\text { MJD } \\
\text { obs } \\
\text { condition } \\
\end{array}$ & $\begin{array}{l}\text { Observation time } \\
\qquad[\mathrm{h}]\end{array}$ & $\begin{array}{c}\text { Significance } \\
\sigma \\
\text { LE: hadr }<0.28 \\
\text { size }>60 \text { phe }\end{array}$ & $\begin{array}{c}\text { Significance FR } \\
\qquad \sigma \\
\text { FR: hadr }<0.16 \\
\text { size }>300 \text { phe }\end{array}$ & $\begin{array}{c}\mathrm{F}(>150 \mathrm{GeV}) \\
\mathrm{cm}^{-2} \mathrm{~s}^{-1}\end{array}$ \\
\hline $57049.176(1)$ & 0.33 & 0.64 & 0.43 & $<3.0 \times 10^{-11}$ \\
\hline $57050.164(1)$ & 0.68 & -0.82 & 0.19 & $<1.4 \times 10^{-11}$ \\
\hline $57067.139(1)$ & 2.05 & 7.98 & 0.20 & $(3.1 \pm 0.6) \times 10^{-11}$ \\
\hline "(1+3) & 2.86 & 7.26 & -0.09 & - \\
\hline "(3) & 0.80 & 0.35 & -0.67 & $<5.0 \times 10^{-11}$ \\
\hline $57068.154(1)$ & 2.53 & 3.19 & 1.16 & $(1.2 \pm 0.5) \times 10^{-11}$ \\
\hline $57069.099(2)$ & 0.32 & -0.04 & 0.42 & $<5.0 \times 10^{-11}$ \\
\hline $57070.147(1)$ & 2.91 & 2.12 & 1.00 & $<2.0 \times 10^{-11}$ \\
\hline $57077.098(1)$ & 0.97 & 2.41 & 1.62 & $<3.5 \times 10^{-11}$ \\
\hline $57082.153(4)$ & 0.94 & 2.79 & -0.50 & $F(>250 G e V)<2.1 \times 10^{-11}$ \\
\hline
\end{tabular}

Notes. Days of observations are listed along with the data qualification (see text for details), length of observation and significance of detection for different analysis cuts. For detections, also the integrated flux above $150 \mathrm{GeV}$ is given. In the instances of nondetection, we provide a $95 \%$ confidence level upper limit.

The MAGIC telescopes are supported by an extensive weather monitor program. Atmospheric transmission at different heights within the MAGIC field of view is obtained with the use of a LIDAR (for details on this see Fruck \& Gaug 2015). For data quality selection we consider the transmission measured at a height of $9 \mathrm{~km}$, with $T_{9 \mathrm{~km}}=1$ representing a perfectly clear sky and $T_{9 \mathrm{~km}}=0$ a complete opacity. MAGIC can carry out observations also during partial moonlight, with the drawback of having a higher energy threshold and larger systematic errors due to a higher contamination from the elevated night sky background (NSB), see Ahnen et al. (2017a). The brightness of the NSB can be monitored from the average current in the camera (DC). S4 0954+65 was observed in a zenith range ranging from $35^{\circ}$ to $50^{\circ}$, for a total of $12.5 \mathrm{~h}$ of data, of which approximtely one hour was lost due to bad weather. In the following we refer to the different observation conditions of our data set as follows:

- good dark data: data taken with dark sky $(D C<1.5 \mu \mathrm{A})$ and good atmospheric condition $\left(T_{9 \mathrm{~km}}>0.85\right)$, used for detection and spectral reconstruction;

- dark data needing atmospheric correction: data taken with dark sky $(D C<1.5 \mu \mathrm{A})$ but under nonoptimal weather conditions $\left(0.55<T_{9 \mathrm{~km}}<0.85\right)$, used for detection and spectral reconstruction after atmospheric correction;

- good low moon data: data taken with elevated NSB due to moonlight $(1.5 \mu<D C<4 \mu \mathrm{A})$ and good atmospheric condition $\left(T_{9 \mathrm{~km}}>0.85\right)$, used only for detection in this particular dataset;

- good moon data: data taken during high NSB due to moonlight $(D C>4 \mu \mathrm{A})$ and good atmospheric condition $\left(T_{9 \mathrm{~km}}>0.85\right)$, used only for detection.

The subsample of dataset selected with condition (1) $(9.48 \mathrm{~h}$ of good quality data) has been analyzed with the standard MAGIC analysis chain (Zanin et al. 2013). The subsample of dataset selected with condition (2) ( $0.32 \mathrm{~h}$ of data) follows the same analysis chain until the estimation of the energy for the events and evaluation of the flux. For this last step, the estimated energy and the effective area are corrected taking into account the enhanced atmospheric absorption (for validation of the procedure see Fruck \& Gaug 2015).

The subsample of dataset selected with condition (3) is applicable only at the day of 14th February, with the first VHE detection. The detection can be claimed from dark data alone (i.e., selected with condition 1 ), but an extra $0.81 \mathrm{~h}$ of data were taken under low moonlight. The data are presented here for completeness, but are not used for spectral reconstruction so as not to increase the systematic error and energy threshold.

The subsample of dataset selected with condition (4) $(0.94 \mathrm{~h}$ of data) requires a special analysis that takes care of the effect of moonlight on data taking, reconstruction and analysis. Details of the procedure can be found in Ahnen et al. (2017a).

The detailed breakdown of significances and estimated VHE fluxes is given in Table A.1. Numbers are presented for the socalled low energy (LE) and full range (FR) cuts. The LE cuts are optimized for an energy range of $E \gtrsim 100 \mathrm{GeV}$ and are particularly appropriate for steep spectrum sources, while FR cuts are optimized for an energy range of $E \gtrsim 250 \mathrm{GeV}$. The cuts are applied on 2 parameters: the "size" parameter, integrated charge (in photoelectrons) in the cleaned shower image; the "hadronness" parameter, computed from the gamma-hadron separation random forest (RF), with a value ranging from zero for the most gamma-like images to one for the most hadronlike images. Indeed the standard MAGIC analysis chain relies on RF techniques to discriminate among gamma and hadronic shower and to better reconstruct the event directions. Lookup tables are used for energy estimation. This is achieved starting from a parametrization of the shower images in the detector. The significance of signal is then calculated with Eq. (17) from Li \& Ma (1983) and using five regions of equal size and distance to the center of camera as the signal region for background estimation. Fluxes are calculated above an energy threshold of $150 \mathrm{GeV}$, which corresponds to the peak of the differential energy distribution of the excess events as a function of estimated event energy. The high energy threshold is due to the high zenith angle of the observation. We note that for data of condition 4, strong moon, we apply an additional minimum cut in the "size" parameter ("size" > 150 phe) of the reconstructed Cherenkov image as prescribed by the moonlight-adapted analysis. This increases the energy threshold to a value of $\sim 250 \mathrm{GeV}$. In case of nondetection, we provide $95 \%$ confidence level upper limits to the flux, calculated following Rolke et al. (2005), considering a systematic error on flux estimation of $30 \%$ (Aleksić et al. 2016). 


\section{Appendix B: Additional VLBA derived parameters}

Table B.1. Time evolution of characteristics of the new radio knots observed from the jet of S4 0954+65.

\begin{tabular}{|c|c|c|c|c|c|c|c|c|}
\hline Epoch & MJD & Flux(Jy) & $x$ & $y$ & $R$ (mas) & $\mathrm{PA}(\mathrm{deg})$ & Size(mas) & Knot \\
\hline \multicolumn{9}{|l|}{$23 \mathrm{Sep} 2014$} \\
\hline 2014.7288 & 56924 & 0.558 & 0.000 & 0.000 & 0.000 & 0.0 & 0.016 & A0 \\
\hline 2014.7288 & 56924 & 0.118 & -0.018 & 0.084 & 0.086 & -12.1 & 0.058 & $\mathrm{~K} 14 \mathrm{~b}$ \\
\hline 2014.7288 & 56924 & 0.160 & -0.077 & 0.289 & 0.300 & -14.9 & 0.066 & K14a \\
\hline 2014.7288 & 56924 & 0.071 & -0.246 & 0.533 & 0.587 & -24.8 & 0.269 & K13 \\
\hline \multicolumn{9}{|l|}{15 Nov 2014} \\
\hline 2014.8740 & 56977 & 0.613 & 0.000 & 0.000 & 0.000 & 0.0 & 0.024 & $\mathrm{~A} 0$ \\
\hline 2014.8740 & 56977 & 0.057 & -0.040 & 0.147 & 0.152 & -15.4 & 0.060 & $\mathrm{~K} 14 \mathrm{~b}$ \\
\hline 2014.8740 & 56977 & 0.089 & -0.094 & 0.336 & 0.349 & -15.7 & 0.077 & $\mathrm{~K} 14 \mathrm{a}$ \\
\hline 2014.8740 & 56977 & 0.025 & -0.328 & 0.576 & 0.663 & -29.7 & 0.226 & K13 \\
\hline \multicolumn{9}{|l|}{5 Dec 2014} \\
\hline 2014.9288 & 56997 & 0.655 & 0.000 & 0.000 & 0.000 & 0.0 & 0.025 & A0 \\
\hline 2014.9288 & 56997 & 0.092 & -0.025 & 0.109 & 0.112 & -12.9 & 0.065 & $\mathrm{~K} 14 \mathrm{~b}$ \\
\hline 2014.9288 & 56997 & 0.105 & -0.094 & 0.319 & 0.332 & -16.4 & 0.069 & K14a \\
\hline 2014.9288 & 56997 & 0.046 & -0.333 & 0.636 & 0.717 & -27.6 & 0.366 & K13 \\
\hline \multicolumn{9}{|l|}{$29 \operatorname{Dec} 2014$} \\
\hline 2014.9945 & 57021 & 0.664 & 0.000 & 0.000 & 0.000 & 0.0 & 0.026 & A0 \\
\hline 2014.9945 & 57021 & 0.079 & -0.084 & 0.267 & 0.280 & -17.5 & 0.105 & $\mathrm{~K} 14 \mathrm{~b}$ \\
\hline 2014.9945 & 57021 & 0.114 & -0.124 & 0.419 & 0.437 & -16.5 & 0.115 & K14a \\
\hline 2014.9945 & 57021 & 0.038 & -0.456 & 0.688 & 0.826 & -33.5 & 0.587 & K13 \\
\hline \multicolumn{9}{|l|}{$14 \mathrm{Feb} 2015$} \\
\hline 2015.1233 & 57067 & 0.899 & 0.000 & 0.000 & 0.000 & 0.0 & 0.021 & A0 \\
\hline 2015.1233 & 57067 & 0.070 & -0.090 & 0.302 & 0.315 & -16.5 & 0.123 & K14a \\
\hline 2015.1233 & 57067 & 0.286 & -0.158 & 0.463 & 0.489 & -18.9 & 0.196 & $\mathrm{~K} 14 \mathrm{~b}$ \\
\hline 2015.1233 & 57067 & 0.031 & -0.426 & 0.759 & 0.870 & -29.3 & 0.420 & K13 \\
\hline \multicolumn{9}{|l|}{11 Apr 2015} \\
\hline 2015.2767 & 57123 & 0.679 & 0.000 & 0.000 & 0.000 & 0.0 & 0.018 & A0 \\
\hline 2015.2767 & 57123 & 0.119 & -0.008 & 0.120 & 0.121 & -3.9 & 0.048 & K15 \\
\hline 2015.2767 & 57123 & 0.111 & -0.099 & 0.368 & 0.381 & -15.0 & 0.110 & $\mathrm{~K} 14 \mathrm{~b}$ \\
\hline 2015.2767 & 57123 & 0.084 & -0.156 & 0.533 & 0.555 & -16.3 & 0.137 & K14a \\
\hline \multicolumn{9}{|l|}{11 May 2015} \\
\hline 2015.3589 & 57153 & 0.354 & 0.000 & 0.000 & 0.000 & 0.0 & 0.028 & A0 \\
\hline 2015.3589 & 57153 & 0.103 & -0.017 & 0.204 & 0.205 & -4.7 & 0.040 & K15 \\
\hline 2015.3589 & 57153 & 0.052 & -0.121 & 0.388 & 0.407 & -17.4 & 0.101 & $\mathrm{~K} 14 \mathrm{~b}$ \\
\hline 2015.3589 & 57153 & 0.084 & -0.177 & 0.568 & 0.595 & -17.3 & 0.195 & K14a \\
\hline \multicolumn{9}{|l|}{9 Jun 2015} \\
\hline 2015.4385 & 57182 & 0.440 & 0.000 & 0.000 & 0.000 & 0.0 & 0.016 & A0 \\
\hline 2015.4385 & 57182 & 0.121 & -0.037 & 0.302 & 0.304 & -6.9 & 0.049 & K15 \\
\hline 2015.4385 & 57182 & 0.050 & -0.166 & 0.458 & 0.487 & -19.9 & 0.112 & $\mathrm{~K} 14 \mathrm{~b}$ \\
\hline 2015.4385 & 57182 & 0.097 & -0.232 & 0.634 & 0.675 & -20.1 & 0.253 & K14a \\
\hline \multicolumn{9}{|l|}{2 Jul 2015} \\
\hline 2015.5014 & 57205 & 0.469 & 0.000 & 0.000 & 0.000 & 0.0 & 0.014 & A0 \\
\hline 2015.5014 & 57205 & 0.092 & -0.050 & 0.360 & 0.363 & -8.0 & 0.051 & K15 \\
\hline 2015.5014 & 57205 & 0.059 & -0.178 & 0.514 & 0.544 & -19.1 & 0.176 & $\mathrm{~K} 14 \mathrm{~b}$ \\
\hline 2015.5014 & 57205 & 0.060 & -0.269 & 0.651 & 0.704 & -22.4 & 0.238 & K14a \\
\hline
\end{tabular}

Notes. For each identified component and for each epoch (i.e., observation), we present: flux, position with respect to core AO, projected size, and position angle.

The detailed information on the time evolution of the radio knot can be found in Table B.1, while the polatization evolution details are shown in Table B.2.
Table B.2. Time evolution of polarization parameters (percentage and angle) for the core A0 observed from the jet of S4 0954+65.

\begin{tabular}{ccc}
\hline \hline MJD & $P \pm d P(\%)$ & EVPA $\pm \mathrm{dE}(\mathrm{deg})$ \\
\hline 56924 & $5.22 \pm 0.77$ & $5.25 \pm 4.23$ \\
56977 & $6.99 \pm 0.80$ & $16.86 \pm 3.28$ \\
56997 & $7.74 \pm 0.72$ & $-16.57 \pm 2.64$ \\
57021 & $8.15 \pm 0.69$ & $-7.33 \pm 2.43$ \\
57067 & $9.78 \pm 0.94$ & $0.31 \pm 2.74$ \\
57123 & $8.52 \pm 0.41$ & $-7.03 \pm 1.37$ \\
57153 & $2.38 \pm 0.83$ & $-7.00 \pm 9.93$ \\
57182 & $3.19 \pm 0.63$ & $-9.34 \pm 5.66$ \\
57205 & $1.06 \pm 0.56$ & $-51.79 \pm 15.3$ \\
\hline
\end{tabular}


Appendix C: Swift-XRT full dataset

Table C.1. S4 0954+65 Swift-XRT exposures.

\begin{tabular}{|c|c|c|c|c|c|c|c|c|}
\hline Date-Time & MJD & $\begin{array}{c}\text { EXP } \\
{[s]} \\
\end{array}$ & $\begin{array}{c}\mathrm{F}(2-10 \mathrm{keV}) \\
{\left[10^{-12}\right]} \\
{\left[\mathrm{erg} \mathrm{cm}^{-2} \mathrm{~s}^{-1}\right]}\end{array}$ & $\begin{array}{c}\mathrm{F}(0.3-10 \mathrm{keV}) \\
{\left[10^{-12}\right]} \\
{\left[\mathrm{erg} \mathrm{cm}^{-2} \mathrm{~s}^{-1}\right]}\end{array}$ & Index & $\chi_{\mathrm{RED}}^{2}$ & DOF & OBSID \\
\hline 2006-07-04T00:49:40 & 53920.04 & 8620.6 & $2.76_{-0.19}^{+0.22}$ & $4.08_{-0.21}^{+0.20}$ & $1.62 \pm 0.06$ & 0.69 & 30 & 00035381001 \\
\hline 2007-03-28T09:06:11 & 54187.38 & 3578.6 & $2.00_{-0.31}^{+0.34}$ & $3.12_{-0.33}^{+0.42}$ & $1.72 \pm 0.12$ & 1.16 & 9 & 00036326001 \\
\hline 2008-01-10T01:09:39 & 54475.05 & 3748.5 & $1.61_{-0.23}^{+0.23}$ & $2.68_{-0.26}^{+0.29}$ & $1.82 \pm 0.11$ & 1.11 & 10 & 00036326002 \\
\hline 2008-01-11T01:20:01 & 54476.06 & 2891.9 & $2.47_{-0.41}^{+0.47}$ & $3.61_{-0.39}^{+0.47}$ & $1.60 \pm 0.12$ & 0.25 & 8 & 00036326003 \\
\hline 2008-01-15T16:10:28 & 54480.67 & 1513.4 & $3.71_{-1.14}^{+1.68}$ & $4.84_{-0.96}^{+1.53}$ & $1.34 \pm 0.23$ & 0.89 & 3 & 00036326004 \\
\hline 2009-01-09T10:57:37 & 54840.46 & 10524.0 & $1.08_{-0.12}^{+0.12}$ & $1.67_{-0.15}^{+0.13}$ & $1.70 \pm 0.08$ & 1.32 & 14 & 00036326005 \\
\hline 2009-11-01T22:49:53 & 55136.95 & 2784.5 & $1.81_{-027}^{+0.42}$ & $2.48_{-0.38}^{+0.37}$ & $1.46 \pm 0.16$ & 0.17 & 4 & 00036326006 \\
\hline 2009-11-05T08:26:28 & 55140.35 & 2906.9 & $1.40_{-0.36}^{+0.51}$ & $2.06_{-0.40}^{+0.42}$ & $1.60 \pm 0.21$ & 1.31 & 2 & 00036326007 \\
\hline 2009-12-12T18:45:25 & 55177.78 & 3848.3 & $3.72_{-0.38}^{+0.39}$ & $4.95_{-0.33}^{+0.35}$ & $1.39 \pm 0.08$ & 0.89 & 15 & 00036326008 \\
\hline 2010-01-23 14:26:34 & 55219.60 & 8873.9 & $3.98_{-0.25}^{+0.25}$ & $5.63_{-0.23}^{+0.28}$ & $1.52 \pm 0.04$ & 1.18 & 48 & 00090100001 \\
\hline 2010-03-12T05:57:53 & 55267.25 & 7980.6 & $3.47_{-0.27}^{+0.22}$ & $4.89_{-0.24}^{+0.19}$ & $1.52 \pm 0.05$ & 1.69 & 38 & 00090100003 \\
\hline 2011-10-13Т04:06:03 & 55847.17 & 1563.3 & $2.95_{-0.75}^{+1.14}$ & $3.95_{-0.76}^{+1.07}$ & $1.40 \pm 0.23$ & 1.56 & 2 & 00036326009 \\
\hline 2011-10-14T13:19:43 & 55848.56 & 3074.2 & $1.67_{-035}^{+0.29}$ & $2.49_{-031}^{+0.38}$ & $1.64 \pm 0.14$ & 1.76 & 6 & 00036326010 \\
\hline 2014-04-28T14:10:59 & 56775.59 & 1540.8 & $2.16_{-0.48}^{+0.43}$ & $3.06_{-0.43}^{+0.52}$ & $1.53 \pm 0.16$ & 1.81 & 3 & 00091892001 \\
\hline 2014-05-28T20:08:46 & 56805.84 & 1920.4 & $5.36_{-0.65}^{+0.74}$ & $7.40_{-0.58}^{+0.64}$ & $1.48 \pm 0.10$ & 1.47 & 12 & 00091892002 \\
\hline 2014-06-25T20:09:39 & 56833.84 & 1670.7 & $1.17_{-0.32}^{+0.51}$ & $2.05_{-0.34}^{+0.50}$ & $1.90 \pm 0.23$ & 0.32 & 2 & 00091892003 \\
\hline 2014-11-17T23:06:57 & 56978.96 & 3262.1 & $12.08_{-0.00}^{+0.95}$ & $15.07_{-0.81}^{+0.74}$ & $1.20 \pm 0.06$ & 1.21 & 32 & 00033530001 \\
\hline 2014-11-22Т13:31:43 & 56983.56 & 4108.0 & $3.74_{-0.29}^{+0.35}$ & $5.59_{-0.33}^{+0.38}$ & $1.64 \pm 0.07$ & 1.65 & 23 & 00033530002 \\
\hline 2015-01-27T19:19:19 & 57049.81 & 1942.9 & $3.89_{-045}^{+0.49}$ & $6.02_{-0.56}^{+0.59}$ & $1.70 \pm 0.10$ & 1.31 & 10 & 00033530003 \\
\hline 2015-02-13T 17:01:10 & 57066.71 & 1962.9 & $11.15_{-0.82}^{+0.84}$ & $18.45_{-0.77}^{+0.92}$ & $1.81 \pm 0.05$ & 1.02 & 41 & 00033530004 \\
\hline 2015-02-15T07:15:48 & 57068.30 & 1893.0 & $10.35_{-0.84}^{+0.02}$ & $14.38_{-0.84}^{+0.84}$ & $1.49 \pm 0.07$ & 1.02 & 24 & 00033530008 \\
\hline 2015-02-16T13:17:09 & 57069.55 & 1905.4 & $16.22_{-1.18}^{+1.06}$ & $22.73_{-1.10}^{+1.12}$ & $1.51 \pm 0.05$ & 0.70 & 39 & 00033530009 \\
\hline 2015-02-17T18:20:49 & 57070.77 & 1775.6 & $21.32_{-1.47}^{+1.42}$ & $31.82_{-1.31}^{+1.67}$ & $1.64 \pm 0.05$ & 1.03 & 36 & 00033530010 \\
\hline 2015-02-18T10:00:55 & 57071.42 & 2092.7 & $14.92_{-0.94}^{+1.17}$ & $20.95_{-1.12}^{+0.97}$ & $1.51 \pm 0.05$ & 1.25 & 36 & 00033530011 \\
\hline 2015-02-19T08:22:52 & 57072.35 & 1071.3 & $13.59_{-1.43}^{+1.23}$ & $19.35_{-1.30}^{+1.41}$ & $1.54 \pm 0.08$ & 0.81 & 18 & 00033530012 \\
\hline 2015-02-20T16:19:13 & 57073.68 & 983.9 & $10.89_{-0.90}^{+1.38}$ & $15.92_{-1.16}^{+1.59}$ & $1.60 \pm 0.09$ & 0.41 & 14 & 00033530013 \\
\hline 2015-02-21T19:29:42 & 57074.81 & 1735.6 & $15.54_{-1.34}^{+1.07}$ & $20.09_{-1.16}^{+1.26}$ & $1.31 \pm 0.06$ & 1.03 & 28 & 00033530014 \\
\hline 2015-02-22T14:41:28 & 57075.61 & 1937.9 & $14.28_{-1.05}^{+1.21}$ & $18.66_{-0.95}^{+1.20}$ & $1.34 \pm 0.05$ & 1.37 & 30 & 00033530015 \\
\hline 2015-02-23Т03:29:19 & 57076.15 & 994.0 & $12.62_{-1.31}^{+1.59}$ & $16.81_{-1.47}^{+1.65}$ & $1.39 \pm 0.09$ & 0.93 & 13 & 00033530017 \\
\hline 2015-02-24T05:07:19 & 57077.21 & 1371.0 & $22.37_{-1.70}^{+1.40}$ & $27.47_{-1.78}^{+2.16}$ & $1.15 \pm 0.06$ & 1.05 & 27 & 00033530018 \\
\hline 2015-03-04T19:34:43 & 57085.82 & 2205.1 & $12.77_{-0.72}^{+0.83}$ & $18.42_{-0.85}^{+0.86}$ & $1.57 \pm 0.05$ & 1.13 & 40 & 00033530019 \\
\hline 2015-03-05Т06:44:02 & 57086.28 & 1578.3 & $16.54_{-1.13}^{+1.55}$ & $20.93_{-1.23}^{+1.53}$ & $1.25 \pm 0.06$ & 1.03 & 24 & 00033530020 \\
\hline 2015-03-06T11:26:12 & 57087.48 & 1875.5 & $7.93_{-0.83}^{+0.83}$ & $10.88_{-1.01}^{+0.85}$ & $1.46 \pm 0.08$ & 0.96 & 18 & 00033530021 \\
\hline 2015-03-07T10:04:13 & 57088.42 & 1311.1 & $9.30_{-1.18}^{+1.19}$ & $14.04_{-1.00}^{+1.22}$ & $1.66 \pm 0.10$ & 0.99 & 13 & 00033530022 \\
\hline 2015-03-08T 14:43:10 & 57089.61 & 1210.7 & $8.69_{-1.82}^{+1.30}$ & $11.41_{-1.42}^{+1.46}$ & $1.35 \pm 0.13$ & 1.13 & 6 & 00033530023 \\
\hline 2015-03-09T16:02:02 & 57090.67 & 1838.0 & $8.47_{-0.95}^{+0.99}$ & $10.76_{-0.78}^{+0.96}$ & $1.26 \pm 0.08$ & 0.67 & 14 & 00033530024 \\
\hline 2015-03-10Т06:27:07 & 57091.27 & 1098.8 & $7.19_{-1.11}^{+1.42}$ & $9.27_{-1.19}^{+1.41}$ & $1.30 \pm 0.13$ & 1.00 & 6 & 00033530025 \\
\hline 2015-03-11T06:22:22 & 57092.27 & 1863.0 & $8.66_{-0.90}^{+0.83}$ & $11.49_{-0.83}^{+0.83}$ & $1.38 \pm 0.07$ & 0.48 & 16 & 00033530026 \\
\hline 2015-06-21T17:29:18 & 57194.73 & 1465.9 & $5.18_{-1.28}^{+1.88}$ & $6.38_{-1.33}^{+1.99}$ & $1.16 \pm 0.24$ & 0.70 & 1 & 00033829001 \\
\hline 2015-06-22Т20:25:32 & 57195.85 & 1965.4 & $3.78_{-0.51}^{+0.78}$ & $5.12_{-0.55}^{+0.58}$ & $1.43 \pm 0.13$ & 0.80 & 6 & 00033829002 \\
\hline 2015-06-24T04:33:29 & 57197.19 & 2202.6 & $2.82_{-0.44}^{+0.56}$ & $4.06_{-0.49}^{+0.01}$ & $1.57 \pm 0.15$ & 0.72 & 6 & 00033829004 \\
\hline 2015-06-25T02:44:56 & 57198.12 & 1635.7 & $4.07_{-1.09}^{+1.25}$ & $5.30_{-1.01}^{+1.26}$ & $1.33 \pm 0.19$ & 0.54 & 4 & 00033829005 \\
\hline 2015-06-26Т01:06:48 & 57199.05 & 986.4 & $4.14_{-1.01}^{+1.16}$ & $5.83_{-0.97}^{+1.49}$ & $1.52 \pm 0.20$ & 1.04 & 3 & 00033829006 \\
\hline 2015-06-27Т04:36:46 & 57200.19 & 1808.0 & $4.19_{-0.55}^{-1.014}$ & $5.70_{-0.72}^{+0.98}$ & $1.44 \pm 0.12$ & 1.10 & 7 & 00033829007 \\
\hline 2015-06-28Т00:54:48 & 57201.04 & 1773.1 & $6.49_{-1.51}^{+1.57}$ & $7.62_{-1.57}^{+1.178}$ & $0.99 \pm 0.19$ & 0.34 & 4 & 00033829008 \\
\hline 2015-06-29T05:54:31 & 57202.25 & 1748.1 & $3.03_{-0.46}^{+0.55}$ & $4.40_{-0.59}^{+0.59}$ & $1.58 \pm 0.13$ & 1.20 & 6 & 00033829009 \\
\hline 2015-06-30T09:00:28 & 57203.38 & 1962.9 & $3.11_{-0.61}^{+0.57}$ & $4.21_{-0.56}^{+0.76}$ & $1.43 \pm 0.15$ & 0.76 & 5 & 00033829010 \\
\hline
\end{tabular}

Notes. For each observation, identified by its date and Swift observation identifier (OBSID), we present: the duration of the exposure (EXP), the integrated energy flux in two energy bands, the best-fit spectral index, the $\chi^{2}$ and degrees of freedom of the fit (DOF).

Table C.1 collects all the analyzed exposures for the Swift-XRT dataset described in Sect. 3. Fluxeshave been extracted from a
20 pixel circular aperture. A different aperture was used on 2015 February 17 (MJD 57070.76), due to pile-up effects. 